\title{
CONVERGENCE OF A CONSTRAINED FINITE ELEMENT DISCRETIZATION OF THE MAXWELL KLEIN GORDON EQUATION*
}

\author{
Snorre H. Christiansen ${ }^{1}$ And Claire Scheid ${ }^{1,2}$
}

\begin{abstract}
As an example of a simple constrained geometric non-linear wave equation, we study a numerical approximation of the Maxwell Klein Gordon equation. We consider an existing constraint preserving semi-discrete scheme based on finite elements and prove its convergence in space dimension 2 for initial data of finite energy.
\end{abstract}

Mathematics Subject Classification. 65M60, 78M10.

Received February 11, 2009. Revised May 21, 2010.

Published online February 21, 2011.

\section{INTRODUCTION}

Non-linear wave equations are at the heart of basic physical models. Fundamental particles are best described by the quantum version of the Yang-Mills-Higgs equation (YMH) and gravitational fields satisfy the Einstein equation for general relativity (GR). For the former the unknown is a connection on a certain vectorbundle over space-time, whereas for the latter it is a pseudo-Riemannian metric. The equations can in both cases be derived from a variational principle involving a Lagrangian with a large gauge-group giving rise to constraints. Second order hyperbolic partial differential equations involving unknowns from differential geometry and stemming from a variational principle will be called geometric wave equations.

The well-posedness of equations with such a rich structure has recently been proved in Sobolev spaces of relatively low regularity. This is relevant both to physics and numerical analysis, since norms related to the energy are the most natural and are most easily incorporated into stability arguments for numerical schemes. For an introduction to the mathematics of geometric wave equations see [28]. Well posedness in the energy norm for the Yang-Mills equation was proved in [21]. For general relativity, progress on the issue is surveyed in $[19]$.

Numerical models exist for both GR and YMH but little, if any, numerical analysis is available for them. The only geometric wave equation for which we are aware of convergence proofs is the wave map equation [3]. With the long-term goal of understanding numerical schemes for GR and YMH we propose to study in this

\footnotetext{
Keywords and phrases. Waves, Maxwell Klein Gordon, non-linear constraints, finite elements, convergence analysis.

* This work, conducted as part of the award "Numerical analysis and simulations of geometric wave equations" made under the European Heads of Research Councils and European Science Foundation EURYI (European Young Investigator) Awards scheme, was supported by funds from the Participating Organizations of EURYI and the EC Sixth Framework Program.

1 CMA c/o Dept. Math, University of Oslo, P.O. Box 1053 Blindern, 0316 Oslo, Norway. snorrec@math.uio.no

${ }^{2}$ Laboratoire Jean Alexandre Dieudonné, Université de Nice Sophia Antipolis, 06108 Nice Cedex 02, France.

Claire.Scheid@unice.fr
} 
paper the simplest equation in the YMH family, namely the Maxwell-Klein-Gordon (MKG) equation obtained with the gauge group $\mathrm{U}(1)$.

In the MKG equation the unknowns are the electromagnetic field, described by a vector potential, and a scalar (complex) field. The scalar field gives rise to a current exciting the electromagnetic field whereas the vector potential enters the coefficients of the wave-equation satisfied by the scalar field. While the wave equation and the Maxwell equations are both linear, the coupling creates a non-linear evolution equation. It is also important that electric charge should be conserved, which gives a non-linear constraint on the flow.

In [10] a finite element method for the YM equation was introduced, whereby the constraint is satisfied through a special application of Lagrange multipliers. In [9] the method was generalized so that it covers all equations in the YMH family and in particular the MKG equation. In this paper we shall prove convergence for this scheme in space dimension 2 with continuous time. The essential features of the scheme are that it preserves energy, which gives control over the curl of the vector potential, and that the constraint preservation gives a weak control over its divergence. Together curl control and divergence control would imply control in the Sobolev space $H^{1}$, if it weren't for the fact that the finite element spaces we use, namely Nédélec's edge elements, are not in this space. Nevertheless we prove a discrete analogue of the Sobolev embedding, valid for Nédélec's edge elements, in the spirit of Kikuchi's compactness result [18]. The proof uses recently constructed commuting interpolators defined on rough functions. Together with Kato's inequality, this gives us strong convergence in $L^{p}$ spaces. A duality argument gives control of the Lagrange multiplier, sufficient to conclude that the limit of the discrete solutions satisfies the continuous MKG equations. The difficulties arising in space dimension 3 are pointed out along the way - the arguments used here fail critically in this case.

The paper is organized as follows. In Section 2, after setting up notations, we give some preliminary results. Since some of the results might be of general interest (in particular the discrete Sobolev embedding), we state and prove them for arbitrary dimension. Section 3 is then dedicated to the exposition of the MKG equation and the semi-discrete scheme we use. Convergence of the approximate solutions of MKG is then obtained in Section 4.

\section{PRELiminaRY}

\subsection{Notations}

Fix an integer $n \geq 2$ and let $\Omega$ be a bounded domain in $\mathbb{R}^{n}$, which is either contractible with a continuously differentiable boundary, or convex. Thus the topology is trivial (curl-free vector fields are gradients) and basic elliptic regularity estimates hold.

\subsubsection{Continuous spaces}

We recall some standard notations. $L^{p}$ spaces:

- $L^{p}(\Omega)$ denotes the classical $L^{p}$ space of real valued functions on $\Omega$, for $p \in[1, \infty]$.

- We say that $\phi \in L^{p}(\Omega, \mathbb{C})$ if $\Re(\phi) \in L^{p}(\Omega)$ and $\Im(\phi) \in L^{p}(\Omega)$, where $\Re$ and $\Im$ are the real and imaginary parts of a complex number. If there is no ambiguity we will sometimes omit the $\mathbb{C}$ from the notation.

Sobolev spaces:

- $H^{s}(\Omega)$ and $H_{0}^{s}(\Omega)$, for $s \in \mathbb{R}$, the usual Hilbertian Sobolev spaces of real valued functions. For $s \in \mathbb{N}$ standard norms and seminorms are denoted $\|\cdot\|_{H^{s}(\Omega)}$ and $|\cdot|_{s}$. For complex valued functions we write $H^{s}(\Omega, \mathbb{C})$.

- $W^{s, p}(\Omega), W_{0}^{s, p}(\Omega)$ denote the Banach spaces obtained by generalizing the above spaces to $L^{p}$ integrability.

- Recall that for $s>0$ and $1<p<\infty, W^{-s, p}(\Omega)$ is dual to $W_{0}^{s, p^{\prime}}(\Omega)$ where $\frac{1}{p}+\frac{1}{p^{\prime}}=1$.

Scalar product:

- We denote by $\cdot$ the canonical scalar product on vectors in $\mathbb{R}^{n}$, and by $|\cdot|$ the associated norm. 
- The real-valued scalar product on $L^{2}(\Omega, \mathbb{C})$ is denoted $\langle\cdot, \cdot\rangle$, and $\|\cdot\|$ is the associated $L^{2}$-norm; $\langle\cdot, \cdot\rangle$ is also used for the duality products between Sobolev spaces with $L^{2}$ as pivot space.

Spaces of vector fields:

- $\mathbf{L}^{2}(\Omega)$ is the space of square integrable vector fields and similar definitions hold for $\mathbf{H}^{1}(\Omega), \mathbf{H}^{s}(\Omega)$, $\mathbf{L}^{p}(\Omega, \mathbb{C})$, etc.

- $\mathbf{H}(\mathbf{c u r l}, \Omega)$ is the space of vector fields in $\mathbb{R}^{n}$ with square integrable curl; the analogue space for the divergence will be noted $\mathbf{H}(\operatorname{div}, \Omega)$. For basic results on $\mathbf{H}(\mathbf{c u r l}, \Omega)$ and $\mathbf{H}(\operatorname{div}, \Omega)$, see $[17,25]$.

- $\mathbf{H}_{0}(\operatorname{curl}, \Omega):=\left\{\mathbf{A} \in \mathbf{H}(\operatorname{curl}, \Omega)\right.$ such that $\gamma_{\tau} \mathbf{A}=0$ on $\left.\partial \Omega\right\}$ where $\gamma_{\tau} \mathbf{A}$ is the tangential component of $\mathbf{A}$ on $\partial \Omega$.

- $\mathbf{V}:=\left\{v \in \mathbf{H}_{0}(\operatorname{curl}, \Omega) \mid \operatorname{div} v=0\right.$ in $\left.\Omega\right\}$.

- For $q \geq 1, \mathbf{H}_{0}^{q}(\operatorname{curl}, \Omega):=\mathbf{H}_{0}(\operatorname{curl}, \Omega) \cap \mathbf{L}^{q}(\Omega)$.

Time dependence. Fix a time $T>0$ :

- For any closed subinterval $I$ of $[0, T]$ and Banach space $X$, we let $\mathcal{C}(I, X)$ be the space of continuous functions from $I$ to $X$, which is a Banach space when equipped with the uniform norm. Also, $\mathcal{C}(0, T ; X)$ will stand for $\mathcal{C}([0, T], X)$.

- For $p \in[1, \infty]$ and $X$ a Banach space, $L^{p}(0, T ; X)$ is the Bochner space defined in [30].

- $\mathcal{C}_{w}(0, T ; X)$ denotes the space of functions of time with values in $X$ which are weakly continuous, explicitly $u \in \mathcal{C}_{w}(0, T ; X)$ means that $t \mapsto l(u(t))$ is continuous on $[0, T]$, for all $l$ in $X^{\star}$, the dual of $X$.

\subsubsection{Semi-discretization}

Let $\left(\mathcal{T}_{h}\right)$ be a regular and quasi-uniform family of simplicial meshes of the domain $\Omega$. As usual the parameter $h$ is also the mesh-width of $\mathcal{T}_{h}$. We use some standard finite element spaces on $\mathcal{T}_{h}$, and simplices are usually denoted by $K$.

- $\mathbb{P}_{1}$ is the space of affine functions (on some open subset of $\mathbb{R}^{n}$ ).

- $Y_{h}^{0}$ is the space of piecewise affine and continuous real functions on $\Omega$, vanishing on the boundary $\partial \Omega[12]$.

- $\mathbf{Y}_{h}^{1}$ is the space of Nédélec edge element vector fields on $\Omega$ (see [4,25]) with vanishing tangential component on the boundary $\partial \Omega$. Thus $\mathbf{Y}_{h}^{1} \subset \mathbf{H}_{0}(\mathbf{c u r l}, \Omega)$.

- $Z_{h}^{0}$ is the space of piecewise affine and continuous complex scalar functions vanishing on the boundary $\partial \Omega$.

- We also put $X_{h}:=\mathbf{Y}_{h}^{1} \times Z_{h}^{0}$.

We define the space of discrete divergence free vectors:

- $\mathbf{V}_{h}:=\left\{v_{h} \in \mathbf{Y}_{h}^{1}:\left\langle v_{h}, \operatorname{grad} \beta_{h}\right\rangle=0, \quad \forall \beta_{h} \in Y_{h}^{0}\right\}$.

Remark 2.1. Many of the difficulties we encounter are related to the fact that:

$$
\mathbf{V}_{h} \nsubseteq \mathbf{V},
$$

but will be resolved by estimating the gap from the former to the latter in appropriate norms.

For the case of a curved boundary we suppose that the mesh and finite element spaces are adapted by appropriate parameterizations, using the techniques in [15].

Throughout the paper, we will use the notation $C$ to refer to a constant independent of $h$. It might be needed to be taken larger in subsequent steps of our arguments, but we nevertheless keep the same notation throughout.

\subsection{Preliminary results}

In this section we present some preliminary results. We state them for arbitrary dimension $n$ and will use them in following sections in the particular case $n=2$, and in remarks concerning $n=3$. These results are either 
quite classical, or generalizations of classical results from the $L^{2}$ case to the $L^{q}$ case, or from time independent fields to time dependent ones.

In the following theorem, where $D$ denotes the standard gradient, we recall Kato's inequality.

Theorem 2.2 ([22]). If $\mathbf{A}: L_{\text {loc }}^{2}\left(\mathbb{R}^{n}, \mathbb{R}^{n}\right), f \in L^{2}\left(\mathbb{R}^{n}, \mathbb{C}\right)$ and $(D+\mathrm{i} \mathbf{A}) f \in L^{2}\left(\mathbb{R}^{n}\right)$, then $|f|$, the modulus of $f$, is in $H^{1}\left(\mathbb{R}^{n}\right)$ and the diamagnetic inequality:

$$
|D| f|(x)| \leq|(D+\mathrm{i} \mathbf{A}) f(x)|
$$

holds pointwise for almost every $x \in \mathbb{R}^{n}$.

The second result we state in this section is the well-known Helmholtz decomposition of fields in $\mathbf{H}(\mathbf{c u r l}, \Omega)$. We have a statement both in the continuous and in the discrete case. Under the hypotheses for our domain, we have in the continuous case:

For every $\boldsymbol{u} \in \mathbf{H}_{0}(\mathbf{c u r l}, \Omega)$, there exists a unique $\stackrel{i}{\boldsymbol{u}} \in \mathbf{V}$ and $p \in H_{0}^{1}(\Omega)$ such that:

$$
\boldsymbol{u}=\stackrel{\imath}{\boldsymbol{u}} \operatorname{grad} p
$$

and in the discrete case:

For every $\boldsymbol{u}_{h} \in \mathbf{Y}_{h}^{1}$, there exists a unique $\stackrel{i}{h}_{h} \in \mathbf{V}_{h}$ and $p_{h} \in Y_{h}^{0}$ such that:

$$
\boldsymbol{u}_{h}=\stackrel{\circ}{\boldsymbol{u}}_{h}+\operatorname{grad} p_{h}
$$

These results can be found for example in $[2,25]$.

In the following many results will rely on Sobolev embeddings [1] one variant of which is recalled here:

Proposition 2.3. For all $q \in] 1, n\left[\right.$, with $q^{\star}$ defined by $1 / q-1 / n=1 / q^{\star}$, one has a continuous embedding $L^{q}(\Omega) \hookrightarrow W^{-1, p}(\Omega)$, for $p \leq q^{\star}$. It is compact when $p<q^{\star}$.

The study of the convergence of the scheme relies on norm estimates in both time and space and on the possibility to extract strongly converging subsequences. Thus we need the characterization of compact sets in the time dependent case, in spaces $L^{\infty}(0, T ; B)$ where $B$ is a Banach space; this has been studied for example by Simon in [30]. The following theorem gives a sufficient condition for compactness for subsets of $L^{\infty}(0, T ; B)$.

Theorem 2.4 ([30]). Suppose that $X, B, Y$ are Banach spaces such that $X \subset B \subset Y$ with continuous embeddings, the first being compact. Suppose $F$ is a bounded set in $L^{\infty}(0, T ; X)$ such that $\frac{\partial F}{\partial t}$ is bounded in $L^{r}(0, T ; Y)$ for some $r>1$. Then $F$ is relatively compact in $\mathcal{C}(0, T ; B)$.

The next propositions (2.5 to 2.12) are generalization of some classical $L^{2}$ results to the $L^{p}$ case (Props. $2.5,2.8$, 2.9 and 2.12) and/or to the time dependent case (Props. 2.7 and 2.11).

The objective of the next two propositions is to establish an analogue of the usual Kikuchi compactness property, in $L^{q}$ and to include time dependence. The property is first proved for fields independent of time, then extended to time dependent fields.

Denote by $2^{*}$ the number such that $\frac{1}{2^{*}}=\frac{1}{2}-\frac{1}{n}$ for $n \geq 2$ (with the convention that $2^{*}=+\infty$ for $n=2$ ). 
Proposition 2.5. Let $1 \leq q \leq 2^{\star}\left(q<2^{\star}\right.$ if $\left.n=2\right)$. Then there exists $C>0$ such that for all $\boldsymbol{v}_{h} \in \mathbf{V}_{h}$,

$$
\left\|\boldsymbol{v}_{h}\right\|_{\mathbf{L}^{q}(\Omega)} \leq C\left\|\operatorname{curl} \boldsymbol{v}_{h}\right\|_{\mathbf{L}^{2}(\Omega)} .
$$

Furthermore if $1 \leq q<2^{*}, \boldsymbol{v}_{h} \in \mathbf{V}_{h}$ and $\mathbf{c u r l} \boldsymbol{v}_{h}$ is bounded in $\mathbf{L}^{2}(\Omega)$, then (out of any subsequence) one can extract a subsequence converging (in norm) in $\mathbf{L}^{q}(\Omega)$ to some $\boldsymbol{v} \in \mathbf{V}$.

Proof. Let $q$ be as in the statement of the theorem. We first prove the bound on the $\mathbf{L}^{q}(\Omega)$ norm.

We denote by:

- $P$ the $\mathbf{L}^{2}$-orthogonal projection on the space of square integrable divergence free vectors. The kernel of $P$ is $\operatorname{grad} H_{0}^{1}$ and this projection preserves the curl, i.e.

$$
\operatorname{curl} \circ P=\operatorname{curl} \text {. }
$$

Furthermore $P \mathbf{V}_{h} \subset \mathbf{V}$.

- $Q_{h}$ the $\mathbf{L}^{p}$ stable projection onto $\mathbf{Y}_{h}^{1}$ constructed in [11] (the ones constructed in [2,26] could also be used). It verifies the following property:

$$
\text { If } \operatorname{curl} \boldsymbol{v}=0 \text {, then } \operatorname{curl} Q_{h} \boldsymbol{v}=0 \text {. }
$$

For $\boldsymbol{v}_{h} \in \mathbf{Y}_{h}^{1}$ we have $P \boldsymbol{v}_{h} \in \mathbf{V}$ and $\operatorname{curl} P \boldsymbol{v}_{h} \in \mathbf{L}^{q}(\Omega)$ so $P \boldsymbol{v}_{h} \in \mathbf{W}^{1, q}(\Omega)$ (using arguments of regularity of solution of elliptic problems, see for example [29]). We get also the estimate:

$$
\left|P \boldsymbol{v}_{h}\right|_{1, q} \leq C\left\|\operatorname{curl} P \boldsymbol{v}_{h}\right\|_{\mathbf{L}^{q}(\Omega)} .
$$

Remark 2.6. We have $\operatorname{curl} \boldsymbol{v}_{h}=\operatorname{curl} P \boldsymbol{v}_{h}=\operatorname{curl} Q_{h} P \boldsymbol{v}_{h}$ for $v_{h} \in \mathbf{Y}_{h}^{1}$.

Choose now $\boldsymbol{v}_{h} \in \mathbf{V}_{h}$.

By triangular inequality,

$$
\left\|\boldsymbol{v}_{h}\right\|_{\mathbf{L}^{q}(\Omega)} \leq\left\|\boldsymbol{v}_{h}-Q_{h} P \boldsymbol{v}_{h}\right\|_{\mathbf{L}^{q}(\Omega)}+\left\|Q_{h} P \boldsymbol{v}_{h}-P \boldsymbol{v}_{h}\right\|_{\mathbf{L}^{q}(\Omega)}+\left\|P \boldsymbol{v}_{h}\right\|_{\mathbf{L}^{q}(\Omega)} .
$$

(a) We have by Bramble-Hilbert type estimates:

$$
\left\|P \boldsymbol{v}_{h}-Q_{h} P \boldsymbol{v}_{h}\right\|_{\mathbf{L}^{q}(\Omega)} \leq C h\left|P \boldsymbol{v}_{h}\right|_{1, q} \leq C h\left\|\operatorname{curl} P \boldsymbol{v}_{h}\right\|_{\mathbf{L}^{q}(\Omega)} \leq C h\left\|\operatorname{curl} \boldsymbol{v}_{h}\right\|_{\mathbf{L}^{q}(\Omega)}
$$

so that we can use the inverse inequality (4.5.11) in [6] and obtain:

$$
\left\|P \boldsymbol{v}_{h}-Q_{h} P \boldsymbol{v}_{h}\right\|_{\mathbf{L}^{q}(\Omega)} \leq C h h^{\min \left(0, \frac{n}{q}-\frac{n}{2}\right)}\left\|\operatorname{curl} \boldsymbol{v}_{h}\right\|_{\mathbf{L}^{2}(\Omega)}
$$

(b) Furthermore

$$
\left\|\boldsymbol{v}_{h}-Q_{h} P \boldsymbol{v}_{h}\right\|_{\mathbf{L}^{q}(\Omega)} \leq C h^{\min \left(0, \frac{n}{q}-\frac{n}{2}\right)}\left\|\boldsymbol{v}_{h}-Q_{h} P \boldsymbol{v}_{h}\right\|_{\mathbf{L}^{2}(\Omega)} .
$$

But since $\operatorname{curl}\left(\boldsymbol{v}_{h}-Q_{h} P \boldsymbol{v}_{h}\right)=0$, we have that $\boldsymbol{v}_{h} \perp \boldsymbol{v}_{h}-Q_{h} P \boldsymbol{v}_{h}$, and $P \boldsymbol{v}_{h} \perp \boldsymbol{v}_{h}-Q_{h} P \boldsymbol{v}_{h}$. So that

$$
\left\|\boldsymbol{v}_{h}-Q_{h} P \boldsymbol{v}_{h}\right\|_{\mathbf{L}^{2}(\Omega)}^{2} \leq\left\|\boldsymbol{v}_{h}-Q_{h} P \boldsymbol{v}_{h}\right\|_{\mathbf{L}^{2}(\Omega)}\left\|P \boldsymbol{v}_{h}-Q_{h} P \boldsymbol{v}_{h}\right\|_{\mathbf{L}^{2}(\Omega)}
$$

Therefore

$$
\left\|\boldsymbol{v}_{h}-Q_{h} P \boldsymbol{v}_{h}\right\|_{\mathbf{L}^{q}(\Omega)} \leq C h^{\min \left(0, \frac{n}{q}-\frac{n}{2}\right)}\left\|P \boldsymbol{v}_{h}-Q_{h} P \boldsymbol{v}_{h}\right\|_{\mathbf{L}^{2}(\Omega)} .
$$

By (2.4), one concludes that

$$
\left\|\boldsymbol{v}_{h}-Q_{h} P \boldsymbol{v}_{h}\right\|_{\mathbf{L}^{q}(\Omega)} \leq C h h^{\min \left(0, \frac{n}{q}-\frac{n}{2}\right)}\left\|\operatorname{curl} \boldsymbol{v}_{h}\right\|_{\mathbf{L}^{2}(\Omega)} .
$$


(c) By the Sobolev embedding of $H^{1}(\Omega)$ into $L^{q}(\Omega)$, we have:

$$
\left\|P \boldsymbol{v}_{h}\right\|_{\mathbf{L}^{q}(\Omega)} \leq C\left\|P \boldsymbol{v}_{h}\right\|_{\mathbf{H}^{1}(\Omega)} .
$$

Friedrich's inequality yields then

$$
\left\|P \boldsymbol{v}_{h}\right\|_{\mathbf{L}^{q}(\Omega)} \leq C\left\|\operatorname{curl} \boldsymbol{v}_{h}\right\|_{\mathbf{L}^{2}(\Omega)} .
$$

(d) Since $1+\frac{n}{q}-\frac{n}{2} \geq 0$, we combine (2.4)-(2.6) to conclude that for some $C>0$ :

$$
\forall \boldsymbol{v}_{h} \in \mathbf{V}_{h}, \quad\left\|\boldsymbol{v}_{h}\right\|_{\mathbf{L}^{q}(\Omega)} \leq C\left\|\operatorname{curl} \boldsymbol{v}_{h}\right\|_{\mathbf{L}^{2}(\Omega)}
$$

It remains to prove that a subsequence of $\boldsymbol{v}_{h}$ converges strongly in $\mathbf{L}^{q}(\Omega)$, if $1 \leq q<2^{*}$.

Since $\left(P \boldsymbol{v}_{h}\right)$ is bounded in $\mathbf{H}^{1}(\Omega)$ we deduce strong convergence in $\mathbf{L}^{q}(\Omega)$ after subsequence extraction. Then since for $1 \leq q<2^{*}$ we have $1+n / q-n / 2>0$, from (2.4) and (2.5) we deduce that $\left(\boldsymbol{v}_{h}\right)$ converges in $\mathbf{L}^{q}(\Omega)$ and has the same limit as $P \boldsymbol{v}_{h}$ in $\mathbf{L}^{q}(\Omega)$. This concludes the proof.

Proposition 2.5 can be generalized to fields with a time dependence:

Proposition 2.7. Let $1<q \leq 2^{*}\left(q<2^{*}\right.$ if $\left.n=2\right)$.

There exists $C>0$ such that for all $\boldsymbol{v}_{h} \in L^{\infty}\left(0, T ; \mathbf{V}_{h}\right)$

$$
\left\|\boldsymbol{v}_{h}\right\|_{L^{\infty}\left(0, T ; \mathbf{L}^{q}(\Omega)\right)} \leq C\left\|\operatorname{curl} \boldsymbol{v}_{h}\right\|_{L^{\infty}\left(0, T ; \mathbf{L}^{2}(\Omega)\right)} .
$$

Furthermore if there exists $C>0$ such that

$$
\left\|\operatorname{curl} \boldsymbol{v}_{h}\right\|_{L^{\infty}\left(0, T ; \mathbf{L}^{2}(\Omega)\right)} \leq C,
$$

and

$$
\left\|\dot{\boldsymbol{v}}_{h}\right\|_{L^{\infty}\left(0, T ; \mathbf{L}^{2}(\Omega)\right)} \leq C,
$$

then for all $q<2^{*}$ there exists $\boldsymbol{v} \in L^{\infty}(0, T ; \mathbf{V})$ such that a subsequence of $\left(\boldsymbol{v}_{h}\right)$ converges in the $L^{\infty}\left(0, T ; \mathbf{L}^{q}(\Omega)\right)$ norm to $\boldsymbol{v}$.

Proof. As all inequalities from the proof of Proposition 2.5 can be transported to time dependent fields, the only point which has to be clarified is that a subsequence of $\boldsymbol{v}_{h}$ has a limit in $L^{\infty}\left(0, T ; \mathbf{L}^{q}(\Omega)\right)$.

Remark that if $\left(\boldsymbol{v}_{h}\right)$ is bounded in $L^{\infty}(0, T ; \mathbf{H}(\mathbf{c u r l}, \Omega))$ then $\left(P \boldsymbol{v}_{h}\right)$ is bounded in $L^{\infty}\left(0, T ; \mathbf{H}^{1}(\Omega)\right)$. Moreover, $P$ commutes with time-differentiation and therefore

$$
\left\|\widehat{\widehat{P \boldsymbol{v}_{h}}}\right\|_{L^{\infty}\left(0, T ; \mathbf{L}^{2}(\Omega)\right)} \leq\left\|\dot{\boldsymbol{v}}_{h}\right\|_{L^{\infty}\left(0, T ; \mathbf{L}^{2}(\Omega)\right)} \leq C,
$$

where $\dot{\overrightarrow{P \boldsymbol{v}_{h}}}$ denotes the time derivative of $P \boldsymbol{v}_{h}$.

Applying Theorem 2.4, $P \boldsymbol{v}_{h}$ converges strongly (considering a subsequence) in $L^{\infty}\left(0, T ; \mathbf{L}^{2}(\Omega)\right.$ ). Using inequalities (2.4) and (2.5) for time dependent fields, $\left\|\boldsymbol{v}_{h}-P \boldsymbol{v}_{h}\right\|_{L^{\infty}\left(0, T ; \mathbf{L}^{2}(\Omega)\right)}$ converges to 0 as $h$ tends to 0 . Then an interpolation inequality between $L^{p}$ spaces, the convergence of $\boldsymbol{v}_{h}$ in $L^{\infty}\left(0, T ; \mathbf{L}^{2}(\Omega)\right)$, and the fact that $\left\|\boldsymbol{v}_{h}\right\|_{L^{\infty}\left(0, T ; \mathbf{L}^{q}(\Omega)\right)}$ is bounded, complete the proof.

The next proposition gives stability results for projections onto finite element spaces. This result will be needed in Section 4.5.

Proposition 2.8. Let $P_{h}^{1}$ be the $\mathbf{L}^{2}$ projection on $\mathbf{Y}_{h}^{1}$ and $P_{h}^{0}$ be the $L^{2}$ projection on $Z_{h}^{0}$. Then:

(a) $P_{h}^{1}$ is stable in $\mathbf{L}^{p}$, and from $\mathbf{H}^{1}(\Omega)$ to $\mathbf{H}(\mathbf{c u r l}, \Omega)$.

(b) $P_{h}^{0}$ is stable in $H^{s}(\Omega)$, for all $-1 \leq s \leq 1$. 
Proof. $\quad$ (a) For $P_{h}^{1}$ :

- Stability in $\mathbf{L}^{p}(\Omega)$

Using the result in [14] in the vectorial case, one obtains stability in the $\mathbf{L}^{p}$-norm.

- Stability in $\mathbf{H}(\mathbf{c u r l}, \Omega)$

Let $Q_{h}$ be the operator constructed in [11] already used in the proof of Proposition 2.5. It is stable both in $\mathbf{L}^{2}(\Omega)$ and $\mathbf{H}(\mathbf{c u r l}, \Omega)$. Using also the inverse inequality between $\mathbf{H}(\mathbf{c u r l}, \Omega)$ and $\mathbf{L}^{2}(\Omega)$, one has:

$$
\begin{aligned}
\forall \boldsymbol{u} \in \mathbf{H}^{1}(\Omega),\left\|P_{h}^{1} \boldsymbol{u}\right\|_{\mathbf{H}(\operatorname{curl}, \Omega)} & \leq\left\|Q_{h} P_{h}^{1} \boldsymbol{u}-Q_{h} \boldsymbol{u}\right\|_{\mathbf{H}(\operatorname{curl}, \Omega)}+\left\|Q_{h} \boldsymbol{u}\right\|_{\mathbf{H}(\operatorname{curl}, \Omega)} \\
& \leq C h^{-1}\left\|Q_{h} P_{h}^{1} \boldsymbol{u}-Q_{h} \boldsymbol{u}\right\|_{\mathbf{L}^{2}(\Omega)}+\left\|Q_{h} \boldsymbol{u}\right\|_{\mathbf{H}(\mathbf{c u r l}, \Omega)} \\
& \leq C h^{-1}\left\|P_{h}^{1} \boldsymbol{u}-\boldsymbol{u}\right\|_{\mathbf{L}^{2}(\Omega)}+\left\|Q_{h} \boldsymbol{u}\right\|_{\mathbf{H}(\mathbf{c u r l}, \Omega)} \\
& \leq C\|\boldsymbol{u}\|_{\mathbf{H}^{1}(\Omega)} .
\end{aligned}
$$

(b) For $P_{h}^{0}$ :

- The stability in $H^{1}(\Omega)$ comes from the result in $[5,13]$.

- The stability in $H^{-1}(\Omega)$ follows by duality.

- The stability in $H^{s}(\Omega)$, for $-1 \leq s \leq 1$ is obtained by using interpolation inequalities.

Discretizing continuous equations leads to discrete ones which should have good convergence properties. The next proposition states this for a particular class of equations.

Proposition 2.9. Let $p \in] 1,+\infty\left[\right.$ be given, $a(\cdot, \cdot)$ the bilinear form on $W^{1, p}(\Omega) \times W^{1, p^{\prime}}(\Omega)\left(1 / p+1 / p^{\prime}=1\right)$ given by:

and for $h>0, f_{h} \in W^{-1, p}(\Omega), f \in W^{-1, p}(\Omega)$.

$$
a(u, v)=\int_{\Omega} \operatorname{grad} u \cdot \operatorname{grad} v,
$$

Let also $u_{h} \in Y_{h}^{0}$ be the solution of

$$
a\left(u_{h}, v_{h}\right)=\left\langle f_{h}, v_{h}\right\rangle, \forall v_{h} \in Y_{h}^{0},
$$

and $u \in W_{0}^{1, p}(\Omega)$ the solution of

Then:

$$
a(u, v)=\langle f, v\rangle, \forall v \in W_{0}^{1, p^{\prime}}(\Omega)
$$

(i) $\left\|u_{h}\right\|_{W^{1, p}(\Omega)} \leq C\left\|f_{h}\right\|_{W^{-1, p}(\Omega)}$.

(ii) If $f_{h} \underset{h \rightarrow 0}{\longrightarrow}$ fin $W^{-1, p}(\Omega)$, then $u_{h} \underset{h \rightarrow 0}{\longrightarrow} u$ in $W_{0}^{1, p}(\Omega)$.

This is essentially a reformulation of the following fact:

Remark 2.10. The bilinear form $a$ verifies a uniform discrete inf-sup condition in the norms $W^{1, p}(\Omega) \times W^{1, p^{\prime}}(\Omega)$ see $[6,29]$.

The following proposition is a generalization to time dependent fields:

Proposition 2.11. Let $T>0$ and $p \in] 1,+\infty\left[\right.$ be given, $a(\cdot, \cdot)$ the bilinear form on $W^{1, p}(\Omega) \times W^{1, p^{\prime}}(\Omega)$ $\left(1 / p+1 / p^{\prime}=1\right)$ given by:

$$
a(u, v)=\int_{\Omega} \operatorname{grad} u \cdot \operatorname{grad} v
$$

and for $h>0, f_{h} \in L^{\infty}\left(0, T ; W^{-1, p}(\Omega)\right), f \in L^{\infty}\left(0, T ; W^{-1, p}(\Omega)\right)$.

Let also $u_{h} \in L^{\infty}\left(0, T ; Y_{h}^{0}\right)$ be the solution of

$$
a\left(u_{h}(t), v_{h}\right)=\left\langle f_{h}(t), v_{h}\right\rangle, \forall v_{h} \in Y_{h}^{0}, \text { for a.e. } t \text { in }[0, T]
$$


and $u \in L^{\infty}\left(0, T ; W_{0}^{1, p}(\Omega)\right)$ the solution of

$$
a(u(t), v)=\langle f(t), v\rangle, \forall v \in W_{0}^{1, p^{\prime}}(\Omega) \text { for a.e. } t \text { in }[0, T] .
$$

Then:

(i) $\left\|u_{h}\right\|_{L^{\infty}\left(0, T ; W^{1, p}(\Omega)\right)} \leq C\left\|f_{h}\right\|_{L^{\infty}\left(0, T ; W^{-1, p}(\Omega)\right)}$.

(ii) If $f_{h} \underset{h \rightarrow 0}{\longrightarrow}$ fin $L^{\infty}\left(0, T ; W^{-1, p}(\Omega)\right)$, then $u_{h} \underset{h \rightarrow 0}{\longrightarrow} u$ in $L^{\infty}\left(0, T ; W_{0}^{1, p}(\Omega)\right)$.

We will also need $\mathbf{L}^{p}$ stability of the Helmholtz decomposition as stated in the following:

Proposition 2.12. The discrete Helmholtz decomposition in $\mathbf{Y}_{h}^{1}$ is stable in $L^{p}$-norm.

Proof. Let $\mathbf{E}_{h} \in \mathbf{Y}_{h}^{1}$ and let $\stackrel{\circ}{\mathbf{E}}_{h} \in \mathbf{V}_{h}$ and $p_{h} \in Y_{h}^{0}$ satisfy $\mathbf{E}_{h}=\stackrel{\circ}{\mathbf{E}}_{h}+\operatorname{grad} p_{h}$. Keeping notations from Proposition 2.9, we deduce that $\operatorname{div} \mathbf{E}_{h} \in W^{-1, p}(\Omega)$, and $a\left(p_{h}, v_{h}\right)=\left\langle\operatorname{div} \mathbf{E}_{h}, v_{h}\right\rangle$ for all $v_{h} \in Y_{h}^{0}$. Therefore:

$$
\left\|p_{h}\right\|_{W^{1, p}(\Omega)} \leq C\left\|\mathbf{E}_{h}\right\|_{\mathbf{L}^{p}(\Omega)} .
$$

Stability of the decomposition follows.

Finally we state a result on compact perturbations (Prop. 2.13) and a result on dual estimates (Prop. 2.14) which we will use in Section 4.5 to get estimates on the time derivative of the discrete solutions and on the Lagrange multiplier.

The following proposition is a generalization of the result obtained in [7], Proposition A.5.2 (see also [8] Thm. 1.12, Cor. 1.17). The dual space of a Banach space $Y$ is denoted $Y^{\star}$.

Proposition 2.13. Let $X$ and $Y$ be two reflexive Banach spaces and $\mathcal{A}: X \rightarrow Y^{*}$ a continuous linear map with closed range. Let $\mathcal{K}$ denote a relatively compact set of compact operators $X \rightarrow Y^{*}$. Let $\left(X_{h}\right)$ and $\left(Y_{h}\right)$ be two families of finite-dimensional subspaces of $X$ and $Y$. Suppose that $\left(Y_{h}\right)$ verifies an approximation property:

$$
\forall y \in Y, \lim _{h \rightarrow 0} \inf _{y^{\prime} \in Y_{h}}\left\|y-y^{\prime}\right\|=0,
$$

that $\mathcal{A}$ satisfies a discrete uniform inf-sup condition on $X_{h} \times Y_{h}$, and for all $\mathcal{B} \in \mathcal{K}, \mathcal{A}+\mathcal{B}$ is injective. Then there exists a constant $C$ such that for all $\mathcal{B} \in \mathcal{K}, \mathcal{A}+\mathcal{B}$ verifies a uniform discrete inf-sup condition with constant $C$.

Proof. We apply Proposition A.5.2 in [7]. For every $\mathcal{B} \in \mathcal{K}$, one can construct a ball $B\left(\mathcal{B}, r_{\mathcal{B}}\right)$ of center $\mathcal{B}$ and radius $r_{\mathcal{B}}$ sufficiently small, such that for $\mathcal{B}^{\prime} \in B\left(\mathcal{B}, r_{\mathcal{B}}\right), \mathcal{A}+\mathcal{B}^{\prime}$ verifies an inf-sup condition independent of $\mathcal{B}^{\prime}$. Denote the corresponding constant by $C_{\mathcal{B}}$. Since $\left\{B\left(\mathcal{B}, r_{\mathcal{B}}\right), \mathcal{B} \in \mathcal{K}\right\}$ covers $\mathcal{K}$, we can extract from it a finite subcover. Let $C$ be the worst inf-sup constant in this finite family. Then for all $\mathcal{B} \in \mathcal{K}, \mathcal{A}+\mathcal{B}$ verifies a uniform inf-sup condition with constant $C$. This concludes the proof.

Proposition 2.14. Let $\mathcal{X}$ and $\mathcal{Y}$ be two Banach spaces equipped with respectively the norms $\|\cdot\|_{\mathcal{X}}$ and $\|\cdot\|_{\mathcal{Y}}$, $a(\cdot, \cdot)$ a continuous bilinear form on $\mathcal{X} \times \mathcal{Y}$.

Then let $\left(\mathcal{X}_{h}\right)$ and $\left(\mathcal{Y}_{h}\right)$ be two families of subspaces of equal finite dimension of $\mathcal{X}$ and $\mathcal{Y}$ respectively. We suppose that $a(\cdot, \cdot)$ verifies a discrete inf-sup condition on $\mathcal{X}_{h} \times \mathcal{Y}_{h}$. We consider $T_{h}: \mathcal{Y}^{\prime} \rightarrow \mathcal{X}_{h}$, such that for all $u \in \mathcal{Y}^{\prime}:$

$$
a\left(T_{h} u, v_{h}\right)=\left\langle u, v_{h}\right\rangle, \forall v_{h} \in \mathcal{Y}_{h},
$$

and $T_{h}^{\prime}: \mathcal{X}^{\prime} \rightarrow \mathcal{Y}_{h}$, such that for all $v \in \mathcal{X}^{\prime}$ :

$$
a\left(u_{h}, T_{h}^{\prime} v\right)=\left\langle u_{h}, v\right\rangle, \forall u_{h} \in \mathcal{X}_{h} .
$$


Let $\mathcal{X}_{+}$and $\mathcal{Y}_{-}$be two other Banach spaces (with respective norms $\|\cdot\|_{\mathcal{X}_{+}}$and $\|\cdot\|_{\mathcal{Y}_{-}}$) such that $\mathcal{X} \subset \mathcal{\mathcal { X } _ { + }}$ and $\mathcal{Y}_{-} \subset \mathcal{Y}$, and suppose that if $v \in \mathcal{X}_{+}^{\prime}$ then $T_{h}^{\prime} v \in \mathcal{Y}_{-}$and one has

$$
\left\|T_{h}^{\prime} v\right\|_{\mathcal{Y}_{-}} \leq C\|v\|_{\mathcal{X}_{+}^{\prime}}
$$

Then for all $u$ in $\mathcal{Y}_{-}^{\prime}$,

$$
\left\|T_{h} u\right\|_{\mathcal{X}_{+}} \leq C\|u\|_{\mathcal{Y}_{-}^{\prime}}
$$

Proof. Existence of solutions is guaranteed by inf-sup conditions. From (2.12)-(2.14), we deduce:

$$
\left\|T_{h} u\right\|_{\mathcal{X}_{+}}=\sup _{v \in \mathcal{X}_{+}^{\prime}} \frac{\left\langle T_{h} u, v\right\rangle}{\|v\|_{\mathcal{X}_{+}^{\prime}}}=\sup _{v \in \mathcal{X}_{+}^{\prime}} \frac{a\left(T_{h} u, T_{h}^{\prime} v\right)}{\|v\|_{\mathcal{X}_{+}^{\prime}}}=\sup _{v \in \mathcal{X}_{+}^{\prime}} \frac{\left\langle u, T_{h}^{\prime} v\right\rangle}{\|v\|_{\mathcal{X}_{+}^{\prime}}} \leq C\|u\|_{\mathcal{Y}_{-}^{\prime}}
$$

This proposition generalizes to the time dependent case in an obvious way.

These preliminary results are valid for arbitrary dimension $n$. From now on we will consider a domain $\Omega$ included in $\mathbb{R}^{2}$, and study the Maxwell Klein Gordon equation in this case. However all along the article the difficulties in dimension $n=3$ will be pointed out.

\section{Equation And Discrete FORMulation}

Let $n=2$ so that $\Omega \subset \mathbb{R}^{2}$.

\subsection{Continuous formulation}

\subsubsection{General setting}

Let $T>0$ be given. Solving the Maxwell Klein Gordon equation consists in finding:

- a time dependent gauge potential defined on $[0, T], t \mapsto \mathcal{A}(t)=\left(\begin{array}{c}\alpha(t) \\ \mathbf{A}(t)\end{array}\right)$ where $\alpha(t)$ is a real function on $\Omega$ and $\mathbf{A}(t)$ a real vector field on $\Omega$; and

- a time dependent complex scalar function on $\Omega$, defined on $[0, T]: t \mapsto \phi(t)$,

which constitute a critical point of the action:

$$
\mathcal{S}(\mathbf{A}, \phi, \alpha)=\frac{1}{2} \int_{0}^{T}\|\operatorname{curl} \mathbf{A}\|_{L^{2}(\Omega)}^{2}-\|\operatorname{grad} \alpha-\dot{\mathbf{A}}\|_{L^{2}(\Omega)}^{2}+\left\|D_{\mathbf{A}} \phi\right\|_{L^{2}(\Omega)}^{2}-\|\dot{\phi}+i \alpha \phi\|_{L^{2}(\Omega)}^{2}
$$

As before $D$ denotes the spatial gradient operator acting on complex functions and $D_{\mathbf{A}} \phi=D \phi+\mathrm{i} \mathbf{A} \phi$ is the covariant derivative of $\phi$.

We can express the variation of $\mathcal{S}$ at $(\alpha, \mathbf{A}, \phi)$ in the direction $\left(\alpha^{\prime}, \mathbf{A}^{\prime}, \phi^{\prime}\right)$. Then the stationarity of the action gives Euler-Lagrange equations:

- variation with respect to $\mathbf{A}^{\prime}$ gives an evolution equation for $\mathbf{A}$,

- variation with respect to $\phi^{\prime}$ gives an evolution equation for $\phi$,

- variation with respect to $\alpha^{\prime}$ gives a constraint on the flow.

For more details on this we refer to [9]. 


\subsubsection{In the temporal gauge}

From now on we turn to the Maxwell Klein Gordon equation in temporal gauge, that is, we impose $\alpha(t) \equiv 0$. The equations to solve on $[0, T]$ are then:

$$
\begin{aligned}
\dot{\mathbf{A}} & =-\mathbf{E} \\
\dot{\phi} & =-\psi, \\
\dot{\mathbf{E}} & =\operatorname{curl}(\operatorname{curl}(\mathbf{A}))+\Im\left(D_{\mathbf{A}} \phi \bar{\phi}\right), \\
\dot{\psi} & =D_{\mathbf{A}}^{*} D_{\mathbf{A}} \phi .
\end{aligned}
$$

The constraint is given by:

$$
\operatorname{div}(\mathbf{E})+\Im(\psi \bar{\phi})=0
$$

We suppose that initial conditions are:

$$
\begin{aligned}
\mathbf{A}(0, .) & =\mathbf{A}^{0}(.) \in \mathbf{H}_{0}(\operatorname{curl}, \Omega) \cap \mathbf{H}^{1}(\Omega) \\
\phi(0, .) & =\phi^{0}(.) \in H_{0}^{1}(\Omega, \mathbb{C}) \\
\mathbf{E}(0, .) & =\mathbf{E}^{0}(.) \in \mathbf{L}^{2}(\Omega), \\
\psi(0, .) & =\psi^{0}(.) \in L^{2}(\Omega, \mathbb{C}),
\end{aligned}
$$

and that they verify the constraint given by $(3.5)$ (in $H^{-1}(\Omega)$ ).

We define the energy of the field at time $t$ by:

$$
\mathcal{H}(t)=\langle\mathbf{E}, \mathbf{E}\rangle+\langle\operatorname{curl} \mathbf{A}, \operatorname{curl} \mathbf{A}\rangle+\langle\psi, \psi\rangle+\left\langle D_{\mathbf{A}} \phi, D_{\mathbf{A}} \phi\right\rangle,
$$

and have that

$$
\mathcal{H}(0)<+\infty
$$

Proposition 3.1. This energy is conserved in time for smooth solutions.

In the rest of the paper we often drop the complex sign $\mathbb{C}$ for simplicity of notation.

Weak solution. We introduce the notion of weak solution to (3.1)-(3.4).

Definition 3.2. (E, A, $\psi, \phi)$ is said to be a weak solution of (3.1)-(3.4), if

- We have:

$-\mathbf{E} \in \mathcal{C}\left(0, T ; \mathbf{H}^{-1}(\Omega)\right) \cap L^{\infty}\left(0, T ; \mathbf{L}^{2}(\Omega)\right)$,

- $\mathbf{A} \in \mathcal{C}\left(0, T ; \mathbf{L}^{2}(\Omega)\right) \cap L^{\infty}\left(0, T ; \mathbf{H}_{0}(\operatorname{curl}, \Omega) \cap \mathbf{W}^{1, q}(\Omega)\right)$ for $q<2$,

$-\psi \in \mathcal{C}\left(0, T ; H^{-1}(\Omega)\right) \cap L^{\infty}\left(0, T ; L^{2}(\Omega)\right)$,

- $\phi \in \mathcal{C}\left(0, T ; L^{2}(\Omega)\right) \cap L^{\infty}\left(0, T ; H_{0}^{1}(\Omega)\right)$.

- $\left\{\begin{aligned} \dot{\mathbf{A}} & =-\mathbf{E}, \\ \dot{\phi} & =-\psi .\end{aligned}\right.$

- For every $\left(\mathbf{E}^{\prime}, \psi^{\prime}\right) \in \mathcal{C}_{c}^{\infty}(] 0, T[\times \Omega)^{2} \times \mathcal{C}_{c}^{\infty}(] 0, T[\times \Omega)$, there holds

$-\int_{0}^{T}\left\langle\mathbf{E}, \dot{\mathbf{E}}^{\prime}\right\rangle \mathrm{d} t-\int_{0}^{T}\left\langle\psi, \dot{\psi}^{\prime}\right\rangle \mathrm{d} t=\int_{0}^{T}\left\langle\operatorname{curl} \mathbf{A}, \operatorname{curl} \mathbf{E}^{\prime}\right\rangle \mathrm{d} t+\int_{0}^{T}\left\langle D_{\mathbf{A}} \phi, \mathrm{i} \phi \mathbf{E}^{\prime}\right\rangle \mathrm{d} t+\int_{0}^{T}\left\langle D_{\mathbf{A}} \phi, D_{\mathbf{A}} \psi^{\prime}\right\rangle \mathrm{d} t$. 


\subsection{Discrete formulation}

\subsubsection{A saddle point problem}

Considering the variational formulation of (3.3) and (3.4), and simply discretizing in space in $X_{h}$ provides a scheme which violates the constraint (3.5). In order to preserve it we consider the following constraint preserving scheme proposed in [9] and formulated as a saddle point problem:

For $T>0$, find $t \mapsto\left(\mathbf{A}_{h}(t), \phi_{h}(t)\right) \in X_{h}$ and a Lagrange multiplier $t \mapsto \beta_{h}(t) \in Y_{h}^{0}$ such that for all $t \in[0, T]$ :

$$
\begin{aligned}
\dot{\mathbf{A}}_{h} & =-\mathbf{E}_{h}, \\
\dot{\phi}_{h} & =-\psi_{h},
\end{aligned}
$$

and for all $\left(\mathbf{E}_{h}^{\prime}, \psi_{h}^{\prime}\right) \in X_{h}$ and all $\beta_{h}^{\prime} \in Y_{h}^{0}$ :

$$
\begin{aligned}
\left\langle\dot{\mathbf{E}}_{h}, \mathbf{E}_{h}^{\prime}\right\rangle+\left\langle\dot{\psi_{h}}, \psi_{h}^{\prime}\right\rangle+ & \left\langle\mathbf{E}_{h}^{\prime}, \operatorname{grad} \beta_{h}\right\rangle-\left\langle\psi_{h}^{\prime}, \mathrm{i} \phi_{h} \beta_{h}\right\rangle=\left\langle\operatorname{curl} \mathbf{A}_{h}, \operatorname{curl} \mathbf{E}_{h}^{\prime}\right\rangle+\left\langle D_{\mathbf{A}_{h}} \phi_{h}, \mathrm{i} \phi_{h} \mathbf{E}_{h}^{\prime}\right\rangle+\left\langle D_{\mathbf{A}_{h}} \phi_{h}, D_{\mathbf{A}_{h}} \psi_{h}^{\prime}\right\rangle, \\
\left\langle\dot{\mathbf{E}}_{h}, \operatorname{grad} \beta_{h}^{\prime}\right\rangle-\left\langle\dot{\psi}_{h}, i \phi_{h} \beta_{h}^{\prime}\right\rangle & =0,
\end{aligned}
$$

with initial conditions:

$$
\begin{aligned}
\mathbf{A}_{h}(0, .) & =\mathbf{A}_{h}^{0} \in \mathbf{Y}_{h}^{1}, \\
\mathbf{E}_{h}(0, .) & =\mathbf{E}_{h}^{0} \in \mathbf{Y}_{h}^{1}, \\
\phi_{h}(0, .) & =\phi_{h}^{0} \in Z_{h}^{0}, \\
\psi_{h}(0, .) & =\psi_{h}^{0} \in Z_{h}^{0},
\end{aligned}
$$

where we suppose that $\mathbf{A}_{h}^{0}, \mathbf{E}_{h}^{0}, \phi_{h}^{0}, \psi_{h}^{0}$ are chosen such that:

(i) $\mathbf{A}_{h}^{0} \underset{h \rightarrow 0}{\longrightarrow} \mathbf{A}^{0}$ in $\mathbf{H}_{0}^{q}(\mathbf{c u r l}, \Omega), \forall q<+\infty$ (for $n \geq 3$ replace by $\left.q \leq 2^{\star}\right)$,

(ii) $\mathbf{E}_{h}^{0} \underset{h \rightarrow 0}{\longrightarrow} \mathbf{E}^{0}$ in $\mathbf{L}^{2}(\Omega)$,

(iii) $\phi_{h}^{0} \underset{h \rightarrow 0}{\longrightarrow} \phi^{0}$ in $H^{1}(\Omega)$,

(iv) $\psi_{h}^{0} \underset{h \rightarrow 0}{\longrightarrow} \psi^{0}$ in $L^{2}(\Omega)$,

and the following constraint is satisfied initially:

$$
\left\langle\mathbf{E}_{h}^{0}, \operatorname{grad} \beta_{h}^{\prime}\right\rangle-\left\langle\psi_{h}^{0}, \mathrm{i} \phi_{h}^{0} \beta_{h}^{\prime}\right\rangle=0, \quad \forall \beta_{h}^{\prime} \in Y_{h}^{0} .
$$

The rationale behind the discrete constraint is as follows. Differentiating (3.5) in time gives:

$$
\operatorname{div}(\dot{\mathbf{E}})+\Im(\dot{\psi} \bar{\phi}+\psi \dot{\bar{\phi}})=0
$$

Then we remark that for solutions:

$$
\Im(\psi \dot{\bar{\phi}})=-\Im(\psi \bar{\psi})=0 .
$$

Finally we remark that for $a \in \mathbb{C}$ and $b \in \mathbb{R}$ we have:

$$
(\Im a) b=\Re(\bar{a} \mathrm{i} b)
$$

so that testing the constraint with a real $\beta^{\prime}$ leads to (3.16).

Notice that it is possible to choose initial conditions satisfying the given constraint. Indeed, one chooses $\phi_{h}^{0}$, $\psi_{h}^{0}$ and $\mathbf{A}_{h}^{0}$ such that (iii), (iv) and (i) are verified. One then considers the Helmholtz decomposition of $\mathbf{E}^{0}$ :

$$
\mathbf{E}^{0}=\stackrel{\circ}{\mathbf{E}}^{0}+\operatorname{grad} p^{0}
$$


Then defining $p_{h}^{0}$ as the solution of $\left\langle\operatorname{grad} p_{h}^{0}, \operatorname{grad} \beta_{h}^{\prime}\right\rangle-\left\langle\psi_{h}^{0}, \mathrm{i} \phi_{h}^{0} \beta_{h}^{\prime}\right\rangle=0$ for all $\beta_{h}^{\prime} \in \mathbf{Y}_{h}^{0}$, one concludes using results from Section 2.2 that $p_{h}^{0}$ converges to $p^{0}$ in $\mathbf{H}^{1}(\Omega)$. Choosing $\stackrel{\circ}{\mathbf{E}}_{h}^{0}$ such that $\stackrel{\circ}{\mathbf{E}}_{h}^{0} \underset{h \rightarrow 0}{\longrightarrow} \stackrel{\circ}{\mathbf{E}^{0}}$ in $\mathbf{L}^{2}(\Omega)$, and defining $\mathbf{E}_{h}^{0}:=\stackrel{\circ}{\mathbf{E}}_{h}^{0}+\operatorname{grad} p_{h}^{0}$ gives the desired property.

\subsubsection{Existence of a solution to the discrete formulation}

The above equation can be viewed with $\left(\mathbf{A}_{h}, \phi_{h}\right) \in X_{h}$ as given parameters. We then can rewrite equations (3.15) and (3.16) as:

$$
\begin{aligned}
\left\langle\dot{\mathbf{E}}_{h}, \mathbf{E}_{h}^{\prime}\right\rangle+\left\langle\dot{\psi_{h}}, \psi_{h}^{\prime}\right\rangle+ & \left\langle\mathbf{E}_{h}^{\prime}, \operatorname{grad} \beta_{h}\right\rangle-\left\langle\psi_{h}^{\prime}, \mathrm{i} \phi_{h} \beta_{h}\right\rangle \\
\left\langle\dot{\mathbf{E}}_{h}, \operatorname{grad} \beta_{h}^{\prime}\right\rangle-\left\langle\dot{\psi}_{h}, \mathrm{i} \phi_{h} \beta_{h}^{\prime}\right\rangle & =0,
\end{aligned}
$$

where

and

$$
f_{\mathbf{A}_{h}, \phi_{h}}\left(\mathbf{E}_{h}^{\prime}\right)=\left\langle\operatorname{curl} \mathbf{A}_{h}, \operatorname{curl} \mathbf{E}_{h}^{\prime}\right\rangle+\left\langle D_{\mathbf{A}_{h}} \phi_{h}, i \phi_{h} \mathbf{E}_{h}^{\prime}\right\rangle
$$

$$
g_{\mathbf{A}_{h}, \phi_{h}}\left(\psi_{h}^{\prime}\right)=\left\langle D_{\mathbf{A}_{h}} \phi_{h}, D_{\mathbf{A}_{h}} \psi_{h}^{\prime}\right\rangle .
$$

Proposition 3.3. Let $h>0$ be fixed. The system given by (3.25) and (3.26) with unknowns $\left(\dot{\mathbf{E}}_{h}, \dot{\psi}_{h}, \beta_{h}\right)$ has a unique solution in $X_{h} \times Y_{h}^{0}$. Furthermore, the solution depends smoothly on the parameters $\left(\mathbf{A}_{h}, \phi_{h}\right)$.

Taking $\mathbf{E}_{h}^{\prime}=\operatorname{grad} \beta_{h}^{\prime}$ and $\psi_{h}^{\prime}=0$ gives the following discrete Babuska-Brezzi compatibility condition:

$$
\inf _{\beta_{h}^{\prime} \in Y_{h}^{0}\left(\mathbf{E}_{h}^{\prime}, \psi_{h}^{\prime}\right) \in X_{h}} \frac{\left\langle\mathbf{E}_{h}^{\prime}, \operatorname{grad} \beta_{h}^{\prime}\right\rangle-\left\langle\psi_{h}^{\prime}, \mathrm{i} \phi_{h} \beta_{h}^{\prime}\right\rangle}{\left(\left|\beta_{h}^{\prime}\right|^{2}+\left|\operatorname{grad} \beta_{h}^{\prime}\right|^{2}\right)^{\frac{1}{2}}\left(\left|\mathbf{E}_{h}^{\prime}\right|^{2}+\left|\psi_{h}^{\prime}\right|^{2}\right)^{\frac{1}{2}}} \geq \frac{1}{C},
$$

where $C$ is a positive constant independent of the time $t$ and of $\phi_{h}$. Since $h$ is fixed and all spaces we are dealing with are of finite dimension and all the considered operators are polynomial in the unknowns, we have proved the proposition.

We denote $\mathcal{P}_{\mathbf{Y}_{h}^{1}}$ the projection from $X_{h} \times Y_{h}^{0}$ on $\mathbf{Y}_{h}^{1}$, and $\mathcal{P}_{Z_{h}^{0}}$ the projection from $X_{h} \times Y_{h}^{0}$ on $Z_{h}^{0}$.

If $\mathcal{S}$ is the solution operator associated to equation (3.25), we are able to solve in $X_{h}$ the equations

$$
\begin{aligned}
\ddot{\mathbf{A}}_{h} & =-\mathcal{P}_{\mathbf{Y}_{h}^{1}} \circ \mathcal{S}\left(\mathbf{A}_{h}, \phi_{h}\right), \\
\ddot{\phi}_{h} & =-\mathcal{P}_{Z_{h}^{0}} \circ \mathcal{S}\left(\mathbf{A}_{h}, \phi_{h}\right),
\end{aligned}
$$

locally in time with initial conditions given by (3.6)-(3.9).

This implies that we have existence of $\left(\mathbf{A}_{h}, \phi_{h}\right) \in X_{h}$ for the discrete formulation locally in time.

We define the discrete energy at any time:

$$
\mathcal{H}_{h}(t)=\frac{1}{2}\left(\left\langle\mathbf{E}_{h}, \mathbf{E}_{h}\right\rangle(t)+\left\langle\operatorname{curl} \mathbf{A}_{h}, \operatorname{curl} \mathbf{A}_{h}\right\rangle(t)+\left\langle\psi_{h}, \psi_{h}\right\rangle(t)+\left\langle D_{\mathbf{A}_{h}} \phi_{h}, D_{\mathbf{A}_{h}} \phi_{h}\right\rangle(t)\right) .
$$

The constraint associated with the discrete formulation of (3.1)-(3.4) is given by:

$$
\left\langle\mathbf{E}_{h}, \operatorname{grad} \beta_{h}^{\prime}\right\rangle-\left\langle\psi_{h}, \mathrm{i} \phi_{h} \beta_{h}^{\prime}\right\rangle=0, \forall \beta_{h}^{\prime} \in Y_{h}^{0} .
$$

One can find in [9] a detailed proof of the following:

Proposition 3.4. Equations (3.13)-(3.16) preserve the constraint (3.29) and the energy of the system.

Energy conservation guarantees that the solutions of equations (3.13)-(3.20) are defined on the whole timeinterval $[0, T]$.

We now would like to prove that the sequence $\left(\mathbf{E}_{h}, \mathbf{A}_{h}, \psi_{h}, \phi_{h}\right)$ converges (in a sense which has to be made precise) to a weak solution of the Maxwell Klein Gordon equation (in the sense of Def. 3.2). 


\section{Convergence of the solution}

The rest of the paper is dedicated to proving the following result:

Theorem 4.1. Let $\mathbf{E}^{0}, \mathbf{A}^{0}, \psi^{0}, \phi^{0}$ be given as in (3.6)-(3.9). There exists:

- $\mathbf{E} \in \mathcal{C}\left(0, T ; \mathbf{H}^{-1}(\Omega)\right) \cap L^{\infty}\left(0, T ; \mathbf{L}^{2}(\Omega)\right)$,

- $\mathbf{A} \in \mathcal{C}\left(0, T ; \mathbf{L}^{2}(\Omega)\right) \cap L^{\infty}\left(0, T ; \mathbf{H}_{0}(\mathbf{c u r l}, \Omega) \cap \mathbf{W}^{1, q}(\Omega)\right)$, for a $q<2$,

- $\psi \in \mathcal{C}\left(0, T ; H^{-1}(\Omega)\right) \cap L^{\infty}\left(0, T ; L^{2}(\Omega)\right)$,

- $\phi \in \mathcal{C}\left(0, T ; L^{2}(\Omega)\right) \cap L^{\infty}\left(0, T ; H_{0}^{1}(\Omega)\right)$,

such that the sequence $\left(\mathbf{E}_{h}, \mathbf{A}_{h}, \psi_{h}, \phi_{h}\right)$ solution of (3.13)-(3.20) converges to $(\mathbf{E}, \mathbf{A}, \psi, \phi)$ with:

- $\mathbf{E}_{h} \underset{h \rightarrow 0}{\longrightarrow} \mathbf{E}$ in $\mathcal{C}\left(0, T ; \mathbf{H}^{-1}(\Omega)\right), \mathbf{E}_{h} \underset{h \rightarrow 0}{\rightarrow} \mathbf{E}$ in $L^{\infty}\left(0, T ; \mathbf{L}^{2}(\Omega)\right)$ weak-star,

- $\mathbf{A}_{h} \underset{h \rightarrow 0}{\longrightarrow} \mathbf{A}$ in $\mathcal{C}\left(0, T ; \mathbf{L}^{q}(\Omega)\right), q<2^{\star}, \operatorname{curl} \mathbf{A}_{h} \underset{h \rightarrow 0}{\rightarrow} \operatorname{curl} \mathbf{A}$ in $L^{\infty}\left(0, T ; \mathbf{L}^{2}(\Omega)\right)$ weak-star,

- $\psi_{h} \underset{h \rightarrow 0}{\longrightarrow} \psi$ in $\mathcal{C}\left(0, T ; H^{-1}(\Omega)\right), \psi_{h} \underset{h \rightarrow 0}{\longrightarrow} \psi$ in $L^{\infty}\left(0, T ; L^{2}(\Omega)\right)$ weak-star,

- $\phi_{h} \underset{h \rightarrow 0}{\longrightarrow} \phi$ in $\mathcal{C}\left(0, T ; L^{q}(\Omega)\right), \forall q<+\infty$, grad $\phi_{h} \underset{h \rightarrow 0}{\rightarrow} \operatorname{grad} \phi$ in $L^{\infty}\left(0, T ; L^{2}(\Omega)\right)$ weak-star.

Furthermore $(\mathbf{E}, \mathbf{A}, \psi, \phi)$ is a weak solution of the Maxwell Klein Gordon equation given by (3.1)-(3.9) in the sense of Definition 3.2 with initial conditions given by (3.6)-(3.9).

Strong convergence in $L^{q}$ spaces of either $\phi_{h}$ or $\mathbf{A}_{h}$ is used to handle the nonlinear terms in the right hand side of (3.12). Energy and constraint preservation give boundedness a priori and some weak convergence properties whereas Sobolev embeddings and the Aubin-Lions lemma give the needed strong convergence.

We first obtain strong convergence for $\phi_{h}$ thanks to a priori estimates on $\mathbf{A}_{h}$ (Sects. 4.1 .2 and 4.2 ). Then Section 4.3 is dedicated to strong convergence on the gauge potential $\mathbf{A}_{h}$. Finally Section 4.5 leads to strong convergence for $\mathbf{E}_{h}, \psi_{h}$ and weak-star convergence on the Lagrange multiplier $\beta_{h}$.

\subsection{A priori estimates}

4.1.1. Bounds in the energy norm

From energy conservation (see Sect. 3.2), we deduce the following bounds

$$
\begin{aligned}
\left\|\mathbf{E}_{h}\right\|_{L^{\infty}\left(0, T ; \mathbf{L}^{2}(\Omega)\right)} & \leq C, \\
\left\|\operatorname{curl} \mathbf{A}_{h}\right\|_{L^{\infty}\left(0, T ; \mathbf{L}^{2}(\Omega)\right)} & \leq C, \\
\left\|\psi_{h}\right\|_{L^{\infty}\left(0, T ; L^{2}(\Omega)\right)} & \leq C, \\
\left\|D_{\mathbf{A}_{h}} \phi_{h}\right\|_{L^{\infty}\left(0, T ; \mathbf{L}^{2}(\Omega)\right)} & \leq C,
\end{aligned}
$$

Kato's inequality, Theorem 2.2 gives:

$$
\left\|D\left|\phi_{h}\right|\right\|_{\mathbf{L}^{2}(\Omega)} \leq C, \text { a.e. in }[0, T],
$$

where $D$ still denotes the standard spatial differentiation operator. And so:

$$
\left\|\left|\phi_{h}\right|\right\|_{L^{\infty}\left(0, T ; H_{0}^{1}(\Omega)\right)} \leq C \text {. }
$$

Using the Sobolev embedding gives for each $q<+\infty$ a bound:

$$
\left\|\phi_{h}\right\|_{L^{\infty}\left(0, T ; L^{q}(\Omega)\right)} \leq C
$$

We deduce some weak convergence properties, after extraction of subsequences. 
(a) It follows from (4.6) that there exists $\phi \in L^{\infty}\left(0, T ; L^{q}(\Omega)\right)$ such that

$$
\phi_{h} \rightarrow \phi \text { as } h \rightarrow 0 \text { in } L^{\infty}\left(0, T ; L^{q}(\Omega)\right) \text { weak-star, for all } q<+\infty \text {. }
$$

By (4.3), we can get also

$$
\dot{\phi}_{h} \rightarrow \dot{\phi} \text { in } L^{\infty}\left(0, T ; L^{2}(\Omega)\right) \text { weak-star. }
$$

(b) In a similar way, from (4.1) we deduce weak-star convergence for $\dot{\mathbf{A}}_{h}$ :

$$
\dot{\mathbf{A}}_{h} \rightarrow \dot{\mathbf{A}} \text { in } L^{\infty}\left(0, T ; \mathbf{L}^{2}(\Omega)\right) \text { weak-star. }
$$

Also, using (4.2), we deduce:

$$
\operatorname{curl} \mathbf{A}_{h} \rightarrow \operatorname{curl} \mathbf{A} \text { in } L^{\infty}\left(0, T ; \mathbf{L}^{2}(\Omega)\right) \text { weak-star. }
$$

In order to prove strong convergence of $\phi_{h}$ we shall first obtain some uniform estimates on $\mathbf{A}_{h}$ in $\mathbf{L}^{q}$ norms, for $q<+\infty$.

\subsubsection{Estimate for the gauge potential $\mathbf{A}_{h}$}

The idea here is to exploit the discrete Helmholtz decomposition of $\mathbf{A}_{h}$ and give uniform estimates for each part of its decomposition.

Uniform estimates on the curl of $\mathbf{A}_{h}$ give estimates on the discrete divergence free part of the gauge potential. In order to have estimates on $\mathbf{A}_{h}$, we will use the constraint which gives some control over the divergence of $\mathbf{A}_{h}$.

The discrete Helmholtz decomposition consists in decomposing the vector field $\mathbf{A}_{h}$ uniquely as the sum of two orthogonal fields (see Sect. 2.2):

$$
\mathbf{A}_{h}(t)=\AA_{h}(t)+\operatorname{grad} p_{h}(t),
$$

where $\AA_{h}(t) \in \mathbf{V}_{h}$, and $p_{h}(t) \in Y_{h}^{0}$ for almost all $t \in[0, T]$.

Estimates on discrete divergence free part. To obtain a uniform estimate in the $\mathbf{L}^{q}$ norm in space $($ for all $q<+\infty)$ of the discrete divergence free part in terms of the $\mathbf{L}^{2}$ norm in space of the curl of the gauge potential $\mathbf{A}_{h}$, we apply the estimate of Proposition 2.7 to $\AA_{h}$ :

Then there holds:

$$
\left\|\AA_{h}\right\|_{L^{\infty}\left(0, T ; \mathbf{L}^{q}(\Omega)\right)} \leq C, \forall q<+\infty .
$$

Remark 4.2. In 3D, we obtain $\left\|\AA_{h}\right\|_{L^{\infty}\left(0, T ; \mathbf{L}^{q}(\Omega)\right)} \leq C, \forall q \leq 6$.

Estimates on the gradient part. The expression of the constraint, if verified at $t=0$, gives us that for all $\beta_{h}^{\prime} \in Y_{h}^{0}$,

$$
\left\langle\dot{\mathbf{A}}_{h}(t), \operatorname{grad} \beta_{h}^{\prime}\right\rangle=\left\langle\dot{\phi}_{h}(t), \mathrm{i} \phi_{h}(t) \beta_{h}^{\prime}\right\rangle \text { for every } t \in[0, T] .
$$

Integrating once more, we obtain

$$
\left\langle\mathbf{A}_{h}(t), \operatorname{grad} \beta_{h}^{\prime}\right\rangle=\left\langle\mathbf{A}_{h}(0), \operatorname{grad} \beta_{h}^{\prime}\right\rangle+\int_{0}^{t}\left\langle\dot{\phi}_{h}, \dot{i} \phi_{h} \beta_{h}^{\prime}\right\rangle .
$$

Using the discrete Helmholtz decomposition, we deduce that

$$
\left\langle\operatorname{grad} p_{h}(t), \operatorname{grad} \beta_{h}^{\prime}\right\rangle=\left\langle f_{h}(t), \beta_{h}^{\prime}\right\rangle,
$$

where

$$
f_{h}(t)=\operatorname{div} \mathbf{A}_{h}^{0}+\int_{0}^{t} \Im\left(\dot{\phi}_{h} \bar{\phi}_{h}\right) .
$$


Lemma 4.3. $f_{h} \in L^{\infty}\left(0, T, W^{-1, q}(\Omega)\right)$ for all $q<+\infty$ and is bounded independently of $h$ in this norm.

Proof. Let $q<+\infty$ be given.

(a) $\operatorname{div} \mathbf{A}_{h}^{0}$ is bounded in $W^{-1, q}(\Omega)$ by construction.

(b) By Section 4.1.1, $\dot{\phi}_{h}$ is bounded in $L^{\infty}\left(0, T ; L^{2}(\Omega)\right),\left|\phi_{h}\right|$ is bounded in $L^{\infty}\left(0, T ; H_{0}^{1}(\Omega)\right)$, so we have: $\dot{\phi}_{h} \bar{\phi}_{h}$ is bounded in $L^{\infty}\left(0, T ; L^{r}(\Omega)\right)$ for all $r<2$.

Put $u_{h}(t)=\int_{0}^{t} \Im\left(\dot{\phi}_{h} \bar{\phi}_{h}\right) \mathrm{d} t$. For each $r<2$ we have a bound $\left\|u_{h}\right\|_{L^{\infty}\left(0, T ; L^{r}(\Omega)\right)} \leq C$.

Using the Sobolev embedding of Proposition 2.3, we deduce that $\left(u_{h}\right)$ is bounded in $L^{\infty}(0, T$; $\left.W^{-1, q}(\Omega)\right)$ for all $q<+\infty$.

(a) and (b) allow us to conclude.

From Proposition 2.9 and the previous lemma, we deduce that for all $q<+\infty$ there exists $C(q)$ such that:

$$
\left\|p_{h}\right\|_{L^{\infty}\left(0, T, W^{1, q}(\Omega)\right)} \leq C(q)
$$

Remark 4.4. In the $3 \mathrm{D}$ case, we have uniform bounds associated with the right-hand sides: $f_{h} \in L^{\infty}(0, T$; $\left.W^{-1, q}(\Omega)\right)$ where $q \leq 3, \dot{\phi}_{h} \bar{\phi}_{h} \in L^{r}(\Omega)$ with $r \leq \frac{3}{2}$, and $p_{h} \in L^{\infty}\left(0, T, W^{1, q}(\Omega)\right)$ for $q \leq 3$.

\subsubsection{Conclusion}

Closing 4.1.1 and 4.1.2 we have:

$$
\left\|\mathbf{A}_{h}\right\|_{L^{\infty}\left(0, T ; \mathbf{L}^{q}(\Omega)\right)} \leq C(q), \forall q<+\infty .
$$

Remark 4.5. In the $3 \mathrm{D}$ case for all $q \leq 3$ we have bounds:

$$
\left\|\mathbf{A}_{h}\right\|_{L^{\infty}\left(0, T ; \mathbf{L}^{q}(\Omega)\right)} \leq C .
$$

\subsection{Strong convergence of $\phi_{h}$}

We shall obtain strong convergence of $\phi_{h}$ in $\mathcal{C}\left(0, T ; L^{q}(\Omega)\right)$ for all $q<+\infty$.

We recall that $D_{\mathbf{A}_{h}} \phi_{h}=D \phi_{h}+\mathrm{i} \mathbf{A}_{h} \phi_{h}$, so that:

$$
\left\|D \phi_{h}\right\|_{L^{\infty}\left(0, T ; \mathbf{L}^{2}(\Omega)\right)} \leq\left\|D_{\mathbf{A}_{h}} \phi_{h}\right\|_{L^{\infty}\left(0, T ; \mathbf{L}^{2}(\Omega)\right)}+\left\|\mathbf{A}_{h} \phi_{h}\right\|_{L^{\infty}\left(0, T ; \mathbf{L}^{2}(\Omega)\right)} .
$$

From estimates (4.12) and (4.6), we can now deduce:

$$
\left\|\phi_{h}\right\|_{L^{\infty}\left(0, T ; H_{0}^{1}(\Omega)\right)} \leq C
$$

Remark 4.6. To have this in 3 dimensions, we use that $\mathbf{A}_{h} \in L^{\infty}\left(0, T ; \mathbf{L}^{3}(\Omega)\right)$ and $\phi_{h} \in L^{\infty}\left(0, T ; L^{6}(\Omega)\right)$ which are the limit cases.

Since $\left\|\dot{\phi}_{h}\right\|_{L^{r}\left(0, T ; L^{2}(\Omega)\right)} \leq C$ for some $r>1$, we deduce by Theorem 2.4 that for a subsequence:

$$
\phi_{h} \rightarrow \phi \text { as } h \rightarrow 0 \text { in } \mathcal{C}\left(0, T ; L^{2}(\Omega)\right)
$$

Then since for all $q<+\infty,\left(\phi_{h}\right)$ is bounded in $L^{\infty}\left(0, T ; L^{q}(\Omega)\right)$, we deduce by an interpolation inequality:

$$
\phi_{h} \rightarrow \phi \text { as } h \rightarrow 0 \text { in } \mathcal{C}\left(0, T ; L^{q}(\Omega)\right)
$$


Remark 4.7. In dimension 3 , we can extract a subsequence $\phi_{h}$ converging to $\phi$ in $\mathcal{C}\left(0, T ; L^{q}(\Omega)\right)$ for all $q<6$.

In order to be able to pass to the limit in equations (3.13)-(3.16), we also need a strong convergence for $\mathbf{A}_{h}$. To do so, we use the discrete Helmholtz decomposition as before and deduce strong convergence separately on the discrete divergence free part and the gradient part.

\subsection{Strong convergence on the gauge potential}

4.3.1. Strong convergence of the discrete divergence free part

We know from energy estimates that:

$$
\left\|\operatorname{curl} \AA_{h}\right\|_{L^{\infty}\left(0, T ; \mathbf{L}^{2}(\Omega)\right)} \leq C,
$$

and from Section 4.1.2:

$$
\left\|\AA_{h}\right\|_{L^{\infty}\left(0, T ; \mathbf{L}^{q}(\Omega)\right)} \leq C .
$$

Since time derivation preserves discrete Helmholtz decomposition, we obtain:

$$
\left\|\frac{\partial}{\partial t} \AA_{h}\right\|_{L^{r}\left(0, T ; \mathbf{L}^{2}(\Omega)\right)} \leq C, \text { for } r>1 .
$$

Therefore, we can apply Proposition 2.7 to $\AA_{h}$ and conclude:

There exists $\AA \in \mathcal{C}\left(0, T ; L^{q}(\Omega)\right) \cap L^{\infty}(0, T ; \mathbf{V})$ such that after extraction of a subsequence, for all $q<+\infty$,

$$
\AA_{h} \underset{h \rightarrow 0}{\longrightarrow} \AA \text { in } \mathcal{C}\left(0, T ; L^{q}(\Omega)\right) .
$$

Remark 4.8. In dimension 3, after extraction of a subsequence, $\AA_{h} \underset{h \rightarrow 0}{\longrightarrow} \AA$ in $\mathcal{C}\left(0, T ; \mathbf{L}^{q}(\Omega)\right)$, for $q<3$.

4.3.2. Strong convergence on the gradient part

We shall now derive strong convergence for grad $p_{h}$ appearing in the discrete Helmholtz decomposition. We keep notations of Section 4.1.2.

Let $f=\operatorname{div} \mathbf{A}^{0}+\int_{0}^{t} \Im(\dot{\phi} \bar{\phi})$, we have $f \in L^{\infty}\left(0, T ; W^{-1, q}(\Omega)\right)$, for $q<+\infty$.

We recall that

$$
u_{h}=\int_{0}^{t} \Im\left(\dot{\phi}_{h} \bar{\phi}_{h}\right) \mathrm{d} t, \text { and }\left\|u_{h}\right\|_{L^{\infty}\left(0, T ; L^{r}(\Omega)\right)} \leq C \text { for } r<2,
$$

and since $\dot{\phi}_{h} \bar{\phi}_{h} \in L^{\infty}\left(0, T ; L^{r}(\Omega)\right), r<2$, bounded independently of $h$, we deduce from Proposition 2.3:

$$
\left\|\dot{u}_{h}\right\|_{L^{\infty}\left(0, T ; W^{-1, q}(\Omega)\right)} \leq C .
$$

From Theorem 2.4, we deduce that there exists $u \in \mathcal{C}\left(0, T ; W^{-1, q}(\Omega)\right)$ such that we can extract a subsequence still denoted $u_{h}$ that converges to $u$ in $\mathcal{C}\left(0, T ; W^{-1, q}(\Omega)\right)$, for all $q<+\infty$. Furthermore, from weak convergence of $\dot{\phi}_{h}$ and strong convergence of $\phi_{h}$ in $L^{\infty}\left(0, T ; L^{2}(\Omega)\right)$, we deduce that $u=\int_{0}^{t} \Im(\dot{\phi} \bar{\phi}) \mathrm{d} t$ on $[0, T]$.

Remark 4.9. In the $3 \mathrm{D}$ case, $u_{h} \rightarrow u$ in $\mathcal{C}\left(0, T ; W^{-1, q}(\Omega)\right), \forall q<3$.

Since $\mathbf{A}_{h}^{0} \underset{h \rightarrow 0}{\longrightarrow} \mathbf{A}^{0}$ in $\mathbf{L}^{q}(\Omega)$ for all $q<+\infty$, we deduce also that up to extraction of a subsequence

$$
f_{h} \underset{h \rightarrow 0}{\longrightarrow} f \text { in } \mathcal{C}\left(0, T ; W^{-1, q}(\Omega)\right), \forall q<+\infty .
$$

Applying Proposition 2.9 yields that there exists $p \in \mathcal{C}\left(0, T ; W_{0}^{1, q}(\Omega)\right)$ such that $p_{h} \underset{h \rightarrow 0}{\longrightarrow} p$ in $\mathcal{C}\left(0, T ; W^{1, q}(\Omega)\right)$. 


\subsubsection{Strong convergence}

The decomposition $\mathbf{A}_{h}=\AA_{h}+\operatorname{grad} p_{h}$ and the last two sections yield, after extraction of subsequences:

$$
\mathbf{A}_{h} \underset{h \rightarrow 0}{\longrightarrow} \mathbf{A}:=\AA+\operatorname{grad} p \text { in } \mathcal{C}\left(0, T ; \mathbf{L}^{q}(\Omega)\right) \text { for all } q<+\infty
$$

Remark 4.10. In 3 dimensions we get $\mathbf{A}_{h} \underset{h \rightarrow 0}{\longrightarrow} \mathbf{A}$ in $\mathcal{C}\left(0, T ; \mathbf{L}^{q}(\Omega)\right)$ for all $q<3$.

\subsection{Conclusion}

To sum up, after extraction of a subsequence we get:

$$
\begin{aligned}
& \mathbf{A}_{h} \underset{h \rightarrow 0}{\longrightarrow} \mathbf{A} \text { in } \mathcal{C}\left(0, T ; \mathbf{L}^{q}(\Omega)\right) \text { for all } q<+\infty \\
& \phi_{h} \underset{h \rightarrow 0}{\longrightarrow} \phi \text { in } \mathcal{C}\left(0, T ; L^{q}(\Omega)\right) \text { for all } q<+\infty
\end{aligned}
$$

We then have strong convergence in $L^{q}$ spaces for both $\phi_{h}$ and $\mathbf{A}_{h}$. We are now looking at some estimates on the second time derivatives of these fields and on the Lagrange multiplier.

\subsection{Estimation by compact perturbation}

Let $q \in] 1,+\infty\left[\right.$ given, and $\mathcal{X}^{q}=\mathbf{L}^{q}(\Omega) \times L^{2}(\Omega) \times W^{1, q}(\Omega)$ equipped with the canonical norm.

Let $\mathbf{a}$ be the bilinear form given by:

$$
\mathbf{a}\left(\mathbf{E}, \psi, \beta ; \mathbf{E}^{\prime}, \psi^{\prime}, \beta^{\prime}\right)=\left\langle\mathbf{E}, \mathbf{E}^{\prime}\right\rangle+\left\langle\psi, \psi^{\prime}\right\rangle+\left\langle\mathbf{E}^{\prime}, \operatorname{grad} \beta\right\rangle+\left\langle\mathbf{E}, \operatorname{grad} \beta^{\prime}\right\rangle,
$$

and $\mathbf{b}_{\phi}$ the one given by:

$$
\mathbf{b}_{\phi}\left(\mathbf{E}, \psi, \beta ; \mathbf{E}^{\prime}, \psi^{\prime}, \beta^{\prime}\right)=-\left\langle\psi^{\prime}, \mathrm{i} \phi \beta\right\rangle-\left\langle\psi, \mathrm{i} \phi \beta^{\prime}\right\rangle .
$$

In the discrete setting, we will denote by $\mathcal{X}_{h}^{q}$ the space $X_{h} \times Y_{h}^{0}$ equipped with the $\mathbf{L}^{q} \times L^{2} \times W^{1, q}$-norm.

Finally $q^{\prime}$ is such that: $\frac{1}{q^{\prime}}+\frac{1}{q}=1$.

\subsubsection{Estimates}

This section is dedicated to the proof of the following:

Proposition 4.11. Fix $q>2$. Let $\left(\dot{E}_{h}, \dot{\psi}_{h}, \beta_{h}\right) \in X_{h} \times Y_{h}^{0}$ be the solution of (3.13)-(3.20), so that

$$
\left(\mathbf{a}+\mathbf{b}_{\phi_{h}}\right)\left(\dot{\mathbf{E}}_{h}, \dot{\psi}_{h}, \beta_{h} ; \mathbf{E}_{h}^{\prime}, \psi_{h}^{\prime}, \beta_{h}^{\prime}\right)=f_{\mathbf{A}_{h}, \phi_{h}}\left(\mathbf{E}_{h}^{\prime}\right)+g_{\mathbf{A}_{h}, \phi_{h}}\left(\psi_{h}^{\prime}\right)
$$

Then $\dot{\mathbf{E}}_{h} \in L^{\infty}\left(0, T ; \mathbf{H}^{-1}(\Omega)\right), \dot{\psi}_{h} \in L^{\infty}\left(0, T ; H^{-1}(\Omega)\right), \beta_{h} \in L^{\infty}\left(0, T ; W^{1, q^{\prime}}(\Omega)\right)$ with uniform bounds:

$$
\begin{aligned}
&\left\|\dot{\mathbf{E}}_{h}\right\|_{L^{\infty}\left(0, T ; \mathbf{H}^{-1}(\Omega)\right)} \leq C, \\
&\left\|\dot{\psi}_{h}\right\|_{L^{\infty}\left(0, T ; H^{-1}(\Omega)\right)} \leq C,
\end{aligned}
$$

and

$$
\left\|\beta_{h}\right\|_{L^{\infty}\left(0, T ; W^{1, q^{\prime}}(\Omega)\right)} \leq C .
$$


Proof. First claim. The bilinear form a is continuous on $\mathcal{X}^{q} \times \mathcal{X}^{q^{\prime}}$ and verifies a uniform discrete inf-sup condition in this norm. This fact is proved in the following two steps:

(a) We have a discrete inf-sup condition on $\left(\beta_{h}, \mathbf{E}_{h}^{\prime}\right) \mapsto\left\langle\mathbf{E}_{h}^{\prime}, \operatorname{grad} \beta_{h}\right\rangle$ :

Indeed grad maps $Y_{h}^{0}$ to $\mathbf{Y}_{h}^{1}$, so we deduce by Remark 2.10 that:

$$
\inf _{\beta_{h} \in Y_{h}^{0}} \sup _{\mathbf{E}_{h}^{\prime} \in \mathbf{Y}_{h}^{1}} \frac{\left\langle\mathbf{E}_{h}^{\prime}, \operatorname{grad} \beta_{h}\right\rangle}{\left\|\beta_{h}\right\|_{W^{1, q}(\Omega)}\left\|\mathbf{E}_{h}^{\prime}\right\|_{\mathbf{L}^{q^{\prime}}(\Omega)}} \geq \frac{1}{C} .
$$

The symmetric inequality (with $q$ and $q^{\prime}$ exchanged) holds also.

(b) We have also a discrete inf-sup condition on the associated kernel:

Indeed as the $L^{2}$ projection is stable in the $L^{q}(\Omega)$-norm (Prop. 2.8), and Helmholtz decomposition is stable in the $L^{q}$-norm (Prop. 2.12), we deduce:

$$
\inf _{\mathbf{E}_{h} \in \mathbf{V}_{h}} \sup _{\mathbf{E}_{h}^{\prime} \in \mathbf{V}_{h}} \frac{\left\langle\mathbf{E}_{h}, \mathbf{E}_{h}^{\prime}\right\rangle}{\left\|\mathbf{E}_{h}\right\|_{\mathbf{L}^{q}(\Omega)}\left\|\mathbf{E}_{h}^{\prime}\right\|_{\mathbf{L}^{q^{\prime}}(\Omega)}} \geq \frac{1}{C}
$$

Furthermore,

$$
\inf _{\psi_{h} \in Z_{h}^{0}} \sup _{\psi_{h}^{\prime} \in Z_{h}^{0}} \frac{\left\langle\psi_{h}, \psi_{h}^{\prime}\right\rangle}{\left\|\psi_{h}\right\|_{L^{2}(\Omega)}\left\|\psi_{h}^{\prime}\right\|_{L^{2}(\Omega)}} \geq \frac{1}{C} .
$$

The following inf-sup condition follows:

$$
\inf _{\left(\mathbf{E}_{h}, \psi_{h}\right) \in \mathbf{V}_{h} \times Z_{h}^{0}\left(\mathbf{E}_{h}^{\prime}, \psi_{h}^{\prime}\right) \in \mathbf{V}_{h} \times Z_{h}^{0}} \frac{\left\langle\mathbf{E}_{h}, \mathbf{E}_{h}^{\prime}\right\rangle+\left\langle\psi_{h}, \psi_{h}^{\prime}\right\rangle}{\left(\left\|\mathbf{E}_{h}\right\|_{\mathbf{L}^{q}(\Omega)}+\left\|\psi_{h}\right\|_{L^{2}(\Omega)}\right)\left(\left\|\mathbf{E}_{h}^{\prime}\right\|_{\mathbf{L}^{q^{\prime}}(\Omega)}+\left\|\psi_{h}^{\prime}\right\|_{L^{2}(\Omega)}\right)} \geq \frac{1}{C} .
$$

This proves the claim.

Furthermore, the space of bilinear forms on $\mathcal{X}^{q} \times \mathcal{X}^{q^{\prime}}$ being equipped with the canonical norm, for any $\phi_{h}$, $\mathbf{b}_{\phi_{h}}$ is a compact bilinear form and $H^{1}(\Omega) \ni \phi \mapsto \mathbf{b}_{\phi}$ is also compact.

From Proposition 2.13, since $\phi_{h}(t)$ is in a bounded subset of $H^{1}(\Omega)$, we deduce that $\mathbf{a}+\mathbf{b}_{\phi_{h}}$ verifies a uniform discrete inf-sup condition independent of $h$ and $t$.

We will now use duality estimates to deduce estimates for solutions $\left(\dot{\mathbf{E}}_{h}, \dot{\psi}_{h}, \beta_{h}\right)$ by applying Proposition 2.14 to special spaces $\mathcal{X}, \mathcal{Y}, \mathcal{X}_{h}, \mathcal{Y}_{h}, \mathcal{X}_{+}, \mathcal{Y}_{-}$.

We define:

$$
\begin{aligned}
\mathcal{X} & =\mathcal{X}^{q^{\prime}}, \\
\mathcal{Y} & =\mathcal{X}^{q}, \\
\mathcal{X}_{h} & =\mathbf{Y}_{h}^{1} \times Z_{h}^{0} \times Y_{h}^{0}=\mathcal{X}_{h}^{q^{\prime}}, \\
\mathcal{Y}_{h} & =\mathbf{Y}_{h}^{1} \times Z_{h}^{0} \times Y_{h}^{0}=\mathcal{X}_{h}^{q}, \\
\mathcal{X}_{+} & =\mathbf{H}^{-1}(\Omega) \times H^{-1}(\Omega) \times W^{1, q^{\prime}}(\Omega), \\
\mathcal{Y}_{-} & =\mathbf{H}_{0}^{q}(\operatorname{curl}, \Omega) \times H_{0}^{1}(\Omega) \times W_{0}^{1, q}(\Omega) .
\end{aligned}
$$

Let $\tilde{\mathbf{a}}=\mathbf{a}+\mathbf{b}_{\phi_{h}}$. For $v=\left(\mathbf{E}_{0}, \psi_{0}, \beta_{0}\right) \in \mathcal{X}_{+}^{\prime}$ define $T_{h}^{\prime} v=\left(\mathbf{E}_{h}^{\prime}, \psi_{h}^{\prime}, \beta_{h}^{\prime}\right) \in \mathcal{Y}_{h}$ to be the solution of:

$$
\tilde{\mathbf{a}}\left(u_{h}, T_{h}^{\prime} v\right)=\left\langle u_{h}, v\right\rangle, \forall u_{h} \in \mathcal{X}_{h} .
$$

We denote any $u_{h} \in \mathcal{X}_{h}$ by $u_{h}=\left(\tilde{\mathbf{E}}_{h}, \tilde{\psi}_{h}, \tilde{\beta}_{h}\right)$. We have $T_{h}^{\prime} v \in \mathcal{Y}_{h}$ so $T_{h}^{\prime} v \in \mathcal{Y}_{-}$. We are looking for a bound on $T_{h}^{\prime} v$ in the space $\mathcal{Y}_{-}$. 
- A bound for $\beta_{h}^{\prime}$ in $W^{1, q}(\Omega)$ is given by the previous uniform discrete inf-sup condition on ã. One obtains:

$$
\left\|\beta_{h}^{\prime}\right\|_{W^{1, q}(\Omega)} \leq C\left(\left\|\mathbf{E}_{0}\right\|_{\mathbf{H}^{1}(\Omega)}+\left\|\psi_{0}\right\|_{H^{1}(\Omega)}+\left\|\beta_{0}\right\|_{W^{-1, q}(\Omega)}\right) .
$$

- One has $\left\langle\psi_{h}^{\prime}, \tilde{\psi}_{h}\right\rangle-\left\langle\tilde{\psi}_{h}, \phi_{h} \beta_{h}^{\prime}\right\rangle=\left\langle\psi_{0}, \tilde{\psi}_{h}\right\rangle$ for all $\tilde{\psi}_{h} \in Z_{h}^{0}$.

The stability for $P_{h}^{0}$, the $L^{2}$ projection, in $H^{-1}(\Omega)$ and $q>2$ give that:

$$
\left\|\psi_{h}^{\prime}\right\|_{H^{1}(\Omega)} \leq C\left(\left\|\phi_{h} \beta_{h}^{\prime}\right\|_{H^{1}(\Omega)}+\left\|\psi_{0}\right\|_{H^{1}(\Omega)}\right) \leq C\left(\left\|\phi_{h}\right\|_{H^{1}(\Omega)}\left\|\beta_{h}^{\prime}\right\|_{W^{1, q}(\Omega)}+\left\|\psi_{0}\right\|_{H^{1}(\Omega)}\right) .
$$

Then using that $\left\|\phi_{h}\right\|_{L^{\infty}\left(0, T ; H^{1}(\Omega)\right)}$ is bounded independently of $h$ and (4.28), we deduce:

$$
\left\|\psi_{h}^{\prime}\right\|_{H^{1}(\Omega)} \leq C\left(\left\|\mathbf{E}_{0}\right\|_{\mathbf{H}^{1}(\Omega)}+\left\|\psi_{0}\right\|_{H^{1}(\Omega)}+\left\|\beta_{0}\right\|_{W^{-1, q}(\Omega)}\right) .
$$

- Furthermore $\left\langle\mathbf{E}_{h}^{\prime}, \tilde{\mathbf{E}}_{h}\right\rangle+\left\langle\tilde{\mathbf{E}}_{h}, \operatorname{grad} \beta_{h}^{\prime}\right\rangle=\left\langle\mathbf{E}_{0}, \tilde{\mathbf{E}}_{h}\right\rangle=\left\langle P_{h}^{1}\left(\mathbf{E}_{0}\right), \tilde{\mathbf{E}}_{h}\right\rangle$ for all $\tilde{\mathbf{E}}_{h} \in \mathbf{Y}_{h}^{1}$.

We have the upper bound of $\mathbf{E}_{h}^{\prime}$ in the $\mathbf{L}^{q}$-norm by the inf-sup condition on $\tilde{\mathbf{a}}$.

Concerning the $L^{2}$-norm of the curl of $\mathbf{E}_{h}^{\prime}$, since $\mathbf{E}_{h}^{\prime}+\operatorname{grad} \beta_{h}^{\prime}=P_{h}^{1}\left(\mathbf{E}_{0}\right)$, we deduce that curl $\mathbf{E}_{h}^{\prime}=$ $\operatorname{curl} P_{h}^{1}\left(\mathbf{E}_{0}\right)$. By the stability of the $L^{2}$ projection from $\mathbf{H}^{1}(\Omega)$ to $\mathbf{H}(\mathbf{c u r l}, \Omega),\left\|\operatorname{curl} \mathbf{E}_{h}^{\prime}\right\|_{\mathbf{L}^{2}(\Omega)} \leq$ We conclude:$$
C\left\|\mathbf{E}_{0}\right\|_{\mathbf{H}^{1}(\Omega)} \text {. And so }\left\|\mathbf{E}_{h}^{\prime}\right\|_{\mathbf{L}^{q}(\Omega)}+\left\|\operatorname{curl} \mathbf{E}_{h}^{\prime}\right\|_{\mathbf{L}^{2}(\Omega)} \leq C\left(\left\|\mathbf{E}_{0}\right\|_{\mathbf{H}^{1}(\Omega)}+\left\|\psi_{0}\right\|_{H^{1}(\Omega)}+\left\|\beta_{0}\right\|_{W^{-1, q}(\Omega)}\right) \text {. }
$$

$$
\left\|\psi_{h}^{\prime}\right\|_{H^{1}(\Omega)}+\left\|\mathbf{E}_{h}^{\prime}\right\|_{\mathbf{L}^{q}(\Omega)}+\left\|\operatorname{curl} \mathbf{E}_{h}^{\prime}\right\|_{\mathbf{L}^{2}(\Omega)}+\left\|\beta_{h}^{\prime}\right\|_{W^{1, q}(\Omega)} \leq C\left(\left\|\psi_{0}\right\|_{H^{1}(\Omega)}+\left\|\mathbf{E}_{0}\right\|_{\mathbf{H}^{1}(\Omega)}+\left\|\beta_{0}\right\|_{W^{-1, q}(\Omega)}\right),
$$

which means that

Let $l_{h}$ be defined by:

$$
\left\|T_{h}^{\prime} v\right\|_{\mathcal{Y}_{-}} \leq\|v\|_{\mathcal{X}_{+}^{\prime}}
$$

$$
\left\langle l_{h},\left(\mathbf{E}^{\prime}, \psi^{\prime}, \beta^{\prime}\right)\right\rangle=\left\langle\operatorname{curl} \mathbf{A}_{h}, \operatorname{curl} \mathbf{E}^{\prime}\right\rangle+\left\langle D_{\mathbf{A}_{h}} \phi_{h}, \phi_{h} \mathbf{E}^{\prime}\right\rangle+\left\langle D_{\mathbf{A}_{h}} \phi_{h}, D_{\mathbf{A}_{h}} \psi^{\prime}\right\rangle
$$

then $\left\|l_{h}\right\|_{L^{\infty}\left(0, T ; \mathcal{Y}_{-}^{\prime}\right)} \leq C$.

We can now use Proposition 2.14 with $u=l_{h}$ to conclude that:

$$
\left\|\dot{\mathbf{E}}_{h}\right\|_{L^{\infty}\left(0, T ; \mathbf{H}^{-1}(\Omega)\right)}+\left\|\dot{\psi}_{h}\right\|_{L^{\infty}\left(0, T ; H^{-1}(\Omega)\right)}+\left\|\beta_{h}\right\|_{L^{\infty}\left(0, T ; W^{1, q^{\prime}}(\Omega)\right)} \leq C .
$$

\subsubsection{Conclusion on the convergence}

- $E_{h}$ is bounded independently of $h$ in $L^{\infty}\left(0, T ; \mathbf{L}^{2}(\Omega)\right)$, and $\dot{E}_{h}$ is bounded independently of $h$ in $L^{\infty}\left(0, T ; \mathbf{H}^{-1}(\Omega)\right)$. It follows from Theorem 2.4 that after extraction of subsequences:

$$
\mathbf{E}_{h}=-\dot{\mathbf{A}}_{h} \rightarrow-\dot{\mathbf{A}} \text { in } \mathcal{C}\left(0, T ; \mathbf{H}^{-s}(\Omega)\right) \text { for all } 0<s \leq 1 .
$$

- A similar conclusion holds for $\psi_{h}$ and $\psi$ :

$$
\psi_{h}=-\dot{\phi}_{h} \rightarrow-\dot{\phi} \text { in } \mathcal{C}\left(0, T ; H^{-s}(\Omega)\right) \text { for all } 0<s \leq 1
$$

- Concerning the Lagrange multiplier $\beta_{h}$, one concludes that there exists $\beta \in L^{\infty}\left(0, T ; W_{0}^{1, q^{\prime}}(\Omega)\right)$ such that:

$$
\beta_{h} \underset{h \rightarrow 0}{\rightarrow} \beta \text { in } L^{\infty}\left(0, T ; W^{1, q^{\prime}}(\Omega)\right) \text { weak-star, }
$$

and by Sobolev embeddings:

$$
\beta_{h} \underset{h \rightarrow 0}{\rightarrow} \beta \text { in } L^{\infty}\left(0, T ; L^{r}(\Omega)\right) \text { weak-star for all } r<\frac{2 q^{\prime}}{2-q^{\prime}} .
$$




\subsection{The limit equation}

We are now able to study the limit of equations (3.13)-(3.20). The convergence obtained on $\phi_{h}$ and $\mathbf{A}_{h}$ permits to take the limit on the right hand side of (3.15). The results obtained in Section 4.5 leads to convergence on the left hand side. A weak convergence on $\beta_{h}$ in the appropriate space is here sufficient due to the strong convergence obtained for $\phi_{h}$. We then deduce that for all $\left(\mathbf{E}^{\prime}, \psi^{\prime}, \beta^{\prime}\right) \in \mathcal{C}_{c}^{\infty}(] 0, T[\times \Omega)^{2} \times \mathcal{C}_{c}^{\infty}(] 0, T[\times \Omega) \times$ $\mathcal{C}_{c}^{\infty}([0, T] \times \Omega)$

$$
\begin{aligned}
\int_{0}^{T}\left\langle\dot{\mathbf{E}}, \mathbf{E}^{\prime}\right\rangle \mathrm{d} t+\int_{0}^{T}\left\langle\dot{\psi}, \psi^{\prime}\right\rangle \mathrm{d} t+\int_{0}^{T}\left\langle\mathbf{E}^{\prime}, \operatorname{grad} \beta\right\rangle \mathrm{d} t-\int_{0}^{T}\left\langle\psi^{\prime}, \mathrm{i} \phi \beta\right\rangle \mathrm{d} t \\
=\int_{0}^{T}\left\langle\operatorname{curl} \mathbf{A}, \operatorname{curl} \mathbf{E}^{\prime}\right\rangle \mathrm{d} t+\int_{0}^{T}\left\langle D_{\mathbf{A}} \phi, \mathrm{i} \phi \mathbf{E}^{\prime}\right\rangle \mathrm{d} t+\int_{0}^{T}\left\langle D_{\mathbf{A}} \phi, D_{\mathbf{A}} \psi^{\prime}\right\rangle \mathrm{d} t
\end{aligned}
$$

and

$$
\int_{0}^{T}\left\langle\dot{\mathbf{E}}, \operatorname{grad} \beta^{\prime}\right\rangle \mathrm{d} t-\int_{0}^{T}\left\langle\dot{\psi}, \mathrm{i} \phi \beta^{\prime}\right\rangle \mathrm{d} t=0
$$

Remark 4.12. This formulation is meaningful since we know from Section 4.5.2 that $\dot{\mathbf{E}} \in L^{\infty}\left(0, T ; \mathbf{H}^{-1}(\Omega)\right)$ and $\dot{\psi} \in L^{\infty}\left(0, T ; H^{-1}(\Omega)\right)$.

Remark 4.13. The convergence on $\mathbf{A}_{h}$ and $\phi_{h}$ obtained in the 3D case (see Rems. 4.7 and 4.10) is insufficient for passing to the limit in the non-linear terms on the right hand side.

Remark 4.14. From (4.34), one deduces that $\operatorname{div} \mathbf{A} \in L^{\infty}\left(0, T ; L^{r}(\Omega)\right)$, with $r<2$, and in consequence $\mathbf{A} \in L^{\infty}\left(0, T ; \mathbf{W}^{1, r}(\Omega)\right)$, with $r<2$.

\subsubsection{Value of the Lagrange multiplier}

One can prove that $\beta$ vanishes as follows. Pick $\beta^{\prime} \in \mathcal{C}_{c}^{\infty}(] 0, T[\times \Omega)$. Due to the regularity in time of the solution, this formulation is also valid almost everywhere on $[0, T]$. We then apply the almost everywhere version of (4.33) to test functions $\mathbf{E}^{\prime}=\operatorname{grad} \beta^{\prime}$ and $\psi^{\prime}=-\mathrm{i} \phi \beta^{\prime}$ and obtain using (4.34):

$$
\left\langle\operatorname{grad} \beta^{\prime}, \operatorname{grad} \beta\right\rangle+\left\langle\phi \beta^{\prime}, \phi \beta\right\rangle=0
$$

Since $\beta \in L^{\infty}\left(0, T ; W_{0}^{1, q^{\prime}}(\Omega)\right),|\phi|^{2} \beta \in L^{\infty}\left(0, T ; L^{2}(\Omega)\right)$ and one then deduces by regularity of solutions of elliptic equations that $\beta \in H_{0}^{1}(\Omega)$. Then we can write:

$$
\langle\operatorname{grad} \beta, \operatorname{grad} \beta\rangle+\langle\phi \beta, \phi \beta\rangle=0,
$$

which gives $\beta \equiv 0$.

\subsubsection{Weak solution of Maxwell Klein Gordon equation}

One concludes that for all $\left(\mathbf{E}^{\prime}, \psi^{\prime}\right) \in \mathcal{C}_{c}^{\infty}(] 0, T[\times \Omega)^{2} \times \mathcal{C}_{c}^{\infty}(] 0, T[\times \Omega)$ :

$$
-\int_{0}^{T}\left\langle\mathbf{E}, \dot{\mathbf{E}}^{\prime}\right\rangle \mathrm{d} t-\int_{0}^{T}\left\langle\psi, \dot{\psi}^{\prime}\right\rangle \mathrm{d} t=\int_{0}^{T}\left\langle\operatorname{curl} \mathbf{A}, \operatorname{curl} \mathbf{E}^{\prime}\right\rangle \mathrm{d} t+\int_{0}^{T}\left\langle D_{\mathbf{A}} \phi, \mathrm{i} \phi \mathbf{E}^{\prime}\right\rangle \mathrm{d} t+\int_{0}^{T}\left\langle D_{\mathbf{A}} \phi, D_{\mathbf{A}} \psi^{\prime}\right\rangle \mathrm{d} t
$$

$(\mathbf{E}, \psi)$ is then a weak solution of Maxwell Klein Gordon equation in the sense of Definition 3.2.

This completes the proof of Theorem 4.1. 


\subsubsection{The question of uniqueness of a weak solution}

The solution $(\mathbf{E}, \mathbf{A}, \psi, \phi)$ obtained verifies equation (4.37), and due to its regularity, following Lemma 8.2 from [23], one obtains:

$$
(\mathbf{E}, \mathbf{A}, \psi, \phi) \in \mathcal{C}_{w}\left(0, T ; \mathbf{L}^{2}(\Omega) \times \mathbf{H}^{s}(\Omega) \times L^{2}(\Omega) \times H^{1}(\Omega)\right) \text { with } s<1
$$

To the best knowledge of the authors, no uniqueness result is available in the literature for this specific regularity and gauge. However, due to the various cases already treated by many authors, we expect uniqueness to hold in this setting. We now discuss some of these results. In [16], uniqueness results are proved, in the entire space and in a bounded domain, in temporal gauge for a class of more regular solutions in $H^{s}, s \geq 2$. Uniqueness for the entire Minkowski space for MKG in the Coulomb gauge for fields in $H^{s}, s \geq 1$ spaces is proved in [20,24]. Unfortunately we only have $s<1$ and change of gauge to the temporal gauge poses the question of the regularity available for the gauge transformation, which is not clear to us. But for the YangMills equation, reputedly harder to solve than the MKG equations, uniqueness has been proved in [31] for the temporal gauge for some $H^{s}, s<1$. Such a result for the case of MKG would be sufficient for our needs. We also notice that in [27] a mixed regularity of the type we obtain (energy control of the electromagnetic field but not full $H^{1}$ control of $\mathbf{A}$ ) is used for uniqueness in Lorenz gauge.

\section{Conclusion}

We have proved that the constraint preserving scheme converges to a weak solution of the Maxwell Klein Gordon equation.

This result leads also to a result of existence of solution with data of finite energy. Unfortunately the proof of convergence does not extend to the three-dimensional case (due to the default of compactness of the Sobolev embedding), as pointed out by the corresponding remarks throughout the paper. But this problem could be investigated in a further work using the notions of concentration compactness and Strichartz estimates.

\section{REFERENCES}

[1] R.A Adams and J.J.F. Fournier, Sobolev Spaces - Pure and Applied Mathematics Series. Second edition, Elsevier (2003).

[2] D.N. Arnold, R.S. Falk and R. Winther, Finite element exterior calculus, homological techniques, and applications. Acta Numer. 15 (2006) 1-155.

[3] S. Bartels, X. Fenga and A. Prohl, Finite element approximations of wave maps into spheres. SIAM J. Numer. Anal. 46 (2007) 61-87.

[4] A. Bossavit, Mixed finite elements and the complex of Whitney forms, in The mathematics of finite elements and applications VI, J. Whiteman Ed., Academic Press, London (1988) 137-144.

[5] J.H. Bramble, J.E. Pasciak and O. Steinbach, On the stability of the $L^{2}$ projection in $H^{1}(\Omega)$. Math. Comput. 71 (2001) $147-156$.

[6] S.C. Brenner and L.R. Scott, The Mathematical Theory of Finite Element Methods. Second edition, Springer (2002).

[7] S.H. Christiansen, Résolution des équations intégrales pour la diffraction d'ondes accoustiques et électromagnétiques. Ph.D. thesis, École polytechnique, France (2002).

[8] S.H. Christiansen, Discrete Fredholm properties and convergence estimates for the Electric Field Integral Equation. Math. Comput. 73 (2004) 143-167.

[9] S.H. Christiansen, Constraint preserving schemes for gauge invariant wave equations. SIAM J. Sci. Comput. 31 (2009) 14481469.

[10] S.H. Christiansen and R. Winther, On constraint preservation in numerical simulations of Yang-Mills equations. SIAM J. Sci. Comput. 28 (2006) 75-101.

[11] S.H. Christiansen and R. Winther, Smoothed projections in finite element exterior calculus. Math. Comput. 77 (2007) 813-829.

[12] P.G. Ciarlet, Basic error estimates for elliptic problems, in Handbook of numerical analysis II, P.G. Ciarlet and J.-L. Lions Eds., North Holland (1991) 17-351.

[13] M. Crouzeix and V. Thomée, The stability in $L_{p}$ and $W_{p}^{1}$ of the $L_{2}$-projection onto finite element function spaces. Math. Comput. 48 (1987) 521-532.

[14] J. Douglas Jr., T. Dupont and L. Wahlbin, The stability in $L^{q}$ of the $L^{2}$-projection into finite element function spaces. Numer. Math. 23 (1975) 193-197. 
[15] F. Dubois, Discrete vector potential representation of a divergence free vector field in three-dimensional domains: Numerical analysis of a model problem. SIAM J. Numer. Anal. 27 (1990) 1103-1141.

[16] J. Ginibre and G. Velo, The Cauchy problem for coupled Yang-Mills and scalar fields in the temporal gauge. Commun. Math. Phys. 82 (1981) 1-28.

[17] V. Girault and P.-A. Raviart, Finite Element approximation of the Navier-Stokes equations. Springer-Verlag, Berlin (1986).

[18] F. Kikuchi, On a discrete compactness property for the Nédélec finite elements. J. Fac. Sci. Univ. Tokyo, Sect. 1 A Math. 36 (1989) 479-490.

[19] S. Klainerman, Mathematical challenges of general relativity. Rend. Mat. Appl. 27 (2007) 105-122.

[20] S. Klainerman and M. Machedon, On the Maxwell-Klein-Gordon equation with finite energy. Duke Math. J. 74 (1994) 19-44.

[21] S. Klainerman and M. Machedon, Finite energy solutions of the Yang-Mills equations in R $\mathbf{R}^{\mathbf{3}+\mathbf{1}}$. Ann. Math. 142 (1995) 39-119.

[22] E.H. Lieb and M. Loss, Analysis Graduate Studies in Mathematics 14. Second edition, AMS (2001).

[23] J.L. Lions and E. Magenes, Problèmes aux limites non homogènes et applications 1. Dunod, Paris (1968).

[24] N. Masmoudi and K. Nakanishi, Uniqueness of Finite Energy solutions for Maxwell-Dirac and Maxwell-Klein-Gordon equations. Commun. Math. Phys. 243 (2003) 123-136.

[25] P. Monk, Finite Element Methods for Maxwell's Equations. Oxford Science Publication (2003).

[26] J. Schöberl, A posteriori error estimates for Maxwell equations. Math. Comput. 77 (2008) 633-649.

[27] S. Selberg and A. Tesfahun, Finite-energy global well-posedness of the Maxwell-Klein-Gordon system in Lorenz gauge. Commun. Partial Differ. Equ. 35 (2010) 1029-1057.

[28] J. Shatah and M. Struwe, Geometric wave equations, Courant Lecture Notes in Mathematics 2. New York University, Courant Institute of Mathematical Sciences, New York, American Mathematical Society, Providence (1998).

[29] C.G. Simader, On Dirichlet Boundary Value Problem. Springer-Verlag (1972).

[30] J. Simon, Compact sets in the space $L^{p}(0, T ; B)$. Ann. Mat. Pura. Appl. 146 (1987) 65-96.

[31] T. Tao, Local well-posedness of the Yang-Mills equation in the temporal gauge below the energy norm. J. Differ. Equ. 189 (2003) 366-382. 